\title{
SUPERSYMMETRY AND SCHRÖDINGER-TYPE OPERATORS WITH DISTRIBUTIONAL MATRIX-VALUED POTENTIALS
}

\author{
JONATHAN ECKHARDT, FRITZ GESZTESY, ROGER NICHOLS, AND GERALD TESCHL
}

\begin{abstract}
Building on work on Miura's transformation by Kappeler, Perry, Shubin, and Topalov, we develop a detailed spectral theoretic treatment of Schrödinger operators with matrix-valued potentials, with special emphasis on distributional potential coefficients.

Our principal method relies on a supersymmetric (factorization) formalism underlying Miura's transformation, which intimately connects the triple of operators $\left(D, H_{1}, H_{2}\right)$ of the form

$$
D=\left(\begin{array}{cc}
0 & A^{*} \\
A & 0
\end{array}\right) \text { in } L^{2}(\mathbb{R})^{2 m} \text { and } H_{1}=A^{*} A, H_{2}=A A^{*} \text { in } L^{2}(\mathbb{R})^{m} .
$$

Here $A=I_{m}(d / d x)+\phi$ in $L^{2}(\mathbb{R})^{m}$, with a matrix-valued coefficient $\phi=$ $\phi^{*} \in L_{\text {loc }}^{1}(\mathbb{R})^{m \times m}, m \in \mathbb{N}$, thus explicitly permitting distributional potential coefficients $V_{j}$ in $H_{j}, j=1,2$, where

$$
H_{j}=-I_{m} \frac{d^{2}}{d x^{2}}+V_{j}(x), \quad V_{j}(x)=\phi(x)^{2}+(-1)^{j} \phi^{\prime}(x), j=1,2 .
$$

Upon developing Weyl-Titchmarsh theory for these generalized Schrödinger operators $H_{j}$, with (possibly, distributional) matrix-valued potentials $V_{j}$, we provide some spectral theoretic applications, including a derivation of the corresponding spectral representations for $H_{j}, j=1,2$. Finally, we derive a local Borg-Marchenko uniqueness theorem for $H_{j}, j=1,2$, by employing the underlying supersymmetric structure and reducing it to the known local Borg-Marchenko uniqueness theorem for $D$.
\end{abstract}

\section{INTRODUCTION}

This paper was inspired by an investigation concerning "the Miura map on the line" by Kappeler, Perry, Shubin, and Topalov [77] in 2005. In it, the authors consider the well-known Miura map,

$$
\phi \mapsto \phi^{2}+\phi^{\prime},
$$

which relates appropriate classes of solutions of the Korteweg-de Vries (KdV) and modified Korteweg-de Vries (mKdV) equation (cf., e.g., 39, 40, 43, 44, and the literature cited therein). The Miura map is closely related with factorizations of the KdV Lax operator $H$, the one-dimensional Schrödinger operator in $L^{2}(\mathbb{R})$, into

Date: October 29, 2018.

2010 Mathematics Subject Classification. Primary 34B20, 34B24, 34L05; Secondary 34B27, 34L10, 34L40.

Key words and phrases. Sturm-Liouville operators, distributional coefficients, WeylTitchmarsh theory, supersymmetry.

Research supported by the Austrian Science Fund (FWF) under Grant No. Y330.

J. Spectral Theory 4, 715-768 (2014). 
a product of two first-order operators of the form

$$
H=A A^{*}=-\frac{d^{2}}{d x^{2}}+V(x), \quad V(x)=\phi(x)^{2}+\phi^{\prime}(x),
$$

where

$$
A=\frac{d}{d x}+\phi(x), \quad A^{*}=-\frac{d}{d x}+\phi(x) .
$$

(While these factorizations are formal at this point, their precise mathematical content is discussed in Sections 2 and 3.) In particular, under the assumption $\phi \in L_{\text {loc }}^{2}(\mathbb{R}), \phi$ real-valued a.e. on $\mathbb{R}$, this permits the authors in [77] to discuss real-valued distributional potentials $V \in H_{\text {loc }}^{-1}(\mathbb{R})$, and hence accomplish a remarkable extension of the standard theory of self-adjoint one-dimensional Schrödinger operators in $L^{2}(\mathbb{R})$ which typically deals with the case of real-valued potentials $V \in L_{\text {loc }}^{1}(\mathbb{R})$ (resp., $\left.V \in L_{\text {loc }}^{2}(\mathbb{R})\right)$. This program is carried out in [77] by relying on oscillation theoretic techniques and Hartman's concept of principal and nonprincipal solutions. In particular, the principal focus of [77] is a detailed investigation of the Miura map (1.1), its range, and its geometry on the real line, with special emphasis on function spaces with low regularity.

As it happens, the Miura map (1.1) is intimately connected with an underlying supersymmetric structure which relates a triple of operators $\left(D, H_{1}, H_{2}\right)$ of the form,

$$
\begin{aligned}
& D=\left(\begin{array}{cc}
0 & A^{*} \\
A & 0
\end{array}\right) \text { in } L^{2}(\mathbb{R})^{2}, \\
& H_{1}=A^{*} A \text { and } H_{2}=A A^{*} \text { in } L^{2}(\mathbb{R}) .
\end{aligned}
$$

Most notably in this context, spectral properties of one of $D, H_{1}, H_{2}$ essentially determine the corresponding spectral properties of the remaining two operators in the triple $\left(D, H_{1}, H_{2}\right)$ (as described in Appendix $\mathrm{A}$ ). In particular, since, in accordance with (1.3), $A=(d / d x)+\phi(x), A^{*}=-(d / d x)+\phi(x)$ deal with the (non-distributional) coefficient $\phi \in L_{\text {loc }}^{2}(\mathbb{R})$ only, so does the Dirac-type operator $D$. Consequently, spectral theory (including Weyl-Titchmarsh theory) for the standard Dirac operator $D$ should lead in an effective and streamlined manner to spectral and Weyl-Titchmarsh theory for the generalized Schrödinger operators $H_{1}$ and $H_{2}$ which may harbor distributional potential coefficients $V_{j}$, as

$$
H_{j}=-\frac{d^{2}}{d x^{2}}+V_{j}(x), \quad V_{j}(x)=\phi(x)^{2}+(-1)^{j} \phi^{\prime}(x), j=1,2 .
$$

Realizing this circle of ideas is precisely what is offered in this paper. Moreover, the fact that exploiting the underlying supersymmetric structure is most natural in this context will become clear as we can effortless incorporate two important generalizations as follows:

- We permit more general coefficients $\phi$ and hence (distributional) coefficients $V_{j}$, $j=1,2$, as we only need to assume $\phi \in L_{\text {loc }}^{1}(\mathbb{R})$.

- We actually consider the matrix-valued case in which $\phi$ and $V_{j}, j=1,2$, are $m \times m$ self-adjoint matrices a.e. on $\mathbb{R}$.

Before describing the content of this paper, it is appropriate to comment on the history of singular Sturm-Liouville operator with special emphasis on the papers devoted to distributional potentials. 
The particular case of point interactions as special distributional coefficients in Schrödinger operators received enormous attention, too numerous to be mentioned here in detail. Hence, we only refer to the standard monographs by Albeverio, Gesztesy, Høegh-Krohn, and Holden [1] and Albeverio and Kurasov [4, and some of the more recent developments in Albeverio, Kostenko, and Malamud [3], Kostenko and Malamud [86, 87]. We also mention the case of discontinuous Schrödinger operators originally considered by Hald 54, motivated by the inverse problem for the torsional modes of the earth. For recent development in this direction we refer to Shahriari, Jodayree Akbarfam, and Teschl [128.

The case of Schrödinger operators with strongly singular and oscillating potentials that should be mentioned in this context goes back to studies by Baeteman and Chadan [7, 8, Combescure [21, Combescure and Ginibre 20, Pearson [112, Rofe-Beketov and Hristov [113, 114 and a more recent contribution treating distributional potentials by Herczyński [56. The case of very general (i.e., threecoefficient) singular Sturm-Liouville operators including distributional potentials has been studied by Bennewitz and Everitt [12] in 1983 (see also [34, Sect. I.2]). They restrict their considerations to compact intervals and focus on the special case of a left-definite setting. An extremely thorough and systematic investigation, including even and odd higher-order operators defined in terms of appropriate quasi-derivatives, and in the general case of matrix-valued coefficients (including distributional potential coefficients in the context of Schrödinger-type operators) was presented by Weidmann 135 in 1987. In fact, the general approach in 12 and [135] draws on earlier discussions of quasi-derivatives in Shin [129]-131, Naimark [11, Ch. V], and Zettl [136. Still, it appears that the distributional coefficients treated in [12 did not catch on and subsequent authors referring to this paper mostly focused on the various left and right-definite aspects developed therein. Similarly, it seems likely that the extraordinary generality exerted by Weidmann 135] in his treatment of higher-order differential operators obscured the fact that he already dealt with distributional potential coefficients back in 1987 .

However, it was not until 1999 that Savchuk and Shkalikov 121 started a new development for Sturm-Liouville (resp., Schrödinger) operators with distributional potential coefficients in connection with areas such as, self-adjointness proofs, spectral and inverse spectral theory, oscillation properties, spectral properties in the non-self-adjoint context, etc. In addition to the important series of papers by Savchuk and Shkalikov [121-[127, we mention other groups such as Albeverio, Hryniv, and Mykytyuk [2, Bak and Shkalikov [9], Ben Amara and Shkalikov [10, Ben Amor and Remling [11, Davies 22, Djakov and Mityagin 24]-27, Eckhardt and Teschl [32, Frayer, Hryniv, Mykytyuk, and Perry [35], Gesztesy and Weikard [48, Goriunov and Mikhailets [51, [52, Hryniv [64, Kappeler and Möhr [76, Kappeler, Perry, Shubin, and Topalov [77, Kappeler and Topalov [78, Hryniv and Mykytyuk [65-72], Hryniv, Mykytyuk, and Perry [73]-[74], Kato [81, Korotyaev [84, [85], Maz'ya and Shaposhnikova [98, Ch. 11], Maz'ya and Verbitsky [99]-102, Mikhailets and Molyboga [103-[107, Mirzoev and Safanova [109, Mykytyuk and Trush [10], Sadovnichaya [116], 117. In particular, the paper by Mirzoev and Safanova [109] is closely related to the present one as it also employs the use of a quasi-derivative of the type $f^{[1]}=f^{\prime}+\phi f$ to define a Schrödinger-type operator via a Miura-type transformation and appears to be the only paper known to us since Weidmann's 1987 monograph and the very recent [108 that deals with the 
matrix-valued case, that is, $f$ is $\mathbb{C}^{m}$-valued, $\phi$ is $\mathbb{C}^{m \times m}$-valued, $m \in \mathbb{N}$. The prime focus of [109] is the computation of deficiency indices of the underlying minimal operator.

It should be mentioned that some of the attraction in connection with distributional potential coefficients in the Schrödinger operator clearly stems from the low-regularity investigations of solutions of the Korteweg-de Vries (KdV) equation. We mention, for instance, Buckmaster and Koch [14, Grudsky and Rybkin [53, Kappeler and Möhr [76, Kappeler and Topalov [79], 80, and Rybkin [115].

The case of strongly singular potentials at an endpoint and the associated WeylTitchmarsh-Kodaira theory for Schrödinger operators can already be found in the seminal paper by Kodaira 83. A gap in Kodaira's approach was later circumvented by Kac [75. The theory did not receive much further attention until it was independently rediscovered and further developed by Gesztesy and Zinchenko [50]. This soon lead to a systematic development of Weyl-Titchmarsh theory for strongly singular potentials and we mention, for instance, Eckhardt 28], Eckhardt and Teschl [31, Fulton 36, Fulton and Langer 37, Fulton, Langer, and Luger 38, Kostenko, Sakhnovich, and Teschl [88, 89, 90, 91, 92, and Kurasov and Luger 94 .

We also mention that a different approach to general (i.e., three-coefficient) singular Sturm-Liouville operators (which are not necessarily assumed to be bounded from below) on an arbitrary interval $(a, b) \subseteq \mathbb{R}$, has been developed simultaneously in [29] in the special scalar case $m=1$. This paper systematically develops Weyl-Titchmarsh theory for differential expressions of the type

$$
\tau f=\frac{1}{r}\left(-\left(p\left[f^{\prime}+\phi f\right]\right)^{\prime}+\phi p\left[f^{\prime}+\phi f\right]+q f\right)
$$

and hence is very close in spirit to the general discussion provided by Weidmann [135. Here the coefficients $p, q, r, \phi$ are real-valued and Lebesgue measurable on $(a, b)$, with $p \neq 0, r>0$ a.e. on $(a, b)$, and $p^{-1}, q, r, \phi \in L_{\text {loc }}^{1}((a, b) ; d x)$, and $f$ is supposed to satisfy

$$
f \in A C_{\mathrm{loc}}((a, b)), p\left[f^{\prime}+\phi f\right] \in A C_{\mathrm{loc}}((a, b)),
$$

with $A C_{\text {loc }}((a, b))$ denoting the set of locally absolutely continuous functions on $(a, b)$. In particular, this study includes distributional coefficients. (The paper [29] does not employ the supersymmetric formalism.) Inverse spectral theory for these operators is treated in [30].

It remains to briefly describe the content of this paper: Section2 2 recalls the basics of Weyl-Titchmarsh theory for supersymmetric Dirac-type operators $D=\left(\begin{array}{cc}0 & A^{*} \\ A & 0\end{array}\right)$ in $L^{2}(\mathbb{R})^{2 m}$, where $A=I_{m}(d / d x)+\phi$ in $L^{2}(\mathbb{R})^{m}$ (cf. (2.3)-(2.6)), with a matrixvalued coefficient $\phi=\phi^{*} \in L_{\text {loc }}^{1}(\mathbb{R})^{m \times m}, m \in \mathbb{N}$, following the treatment in [16. In particular, we review Weyl-Titchmarsh theory for $D$ on the half-line and the full real line, including the $2 m \times 2 m$ matrix-valued Green's function of $D$. In Section 3 we exploit the supersymmetric structure of $D$ and analyze the underlying generalized Schrödinger operators $H_{1}=A^{*} A$ and $H_{2}=A A^{*}$ in $L^{2}(\mathbb{R})^{m}$. We derive the Weyl-Titchmarsh solutions for $H_{j}, j=1,2$, given those of $D$ described in Section 2, and describe the precise connection between the half-line Weyl-Titchmarsh matrices of $H_{j}, j=1,2$, and $D$. In addition, we construct the $m \times m$ matrix-valued Green's functions of $H_{j}, j=1,2$, and the corresponding analogs belonging to the half-lines $\left[x_{0}, \infty\right)$ and $\left(-\infty, x_{0}\right]$ with a Dirichlet boundary condition at $x_{0}$. In our 
final Section 4 we provide some spectral theoretic applications of the supersymmetric approach outlined in Section 3 and after deriving the fundamental aspects of Weyl-Titchmarsh theory for the generalized Schrödinger operators $H_{j}, j=1,2$, and a discussion of the corresponding spectral representations, we derive a local Borg-Marchenko uniqueness theorem by utilizing the known analog for the Dirac operator $D$. Supersymmetric Dirac-type operators and associated commutation methods are briefly summarized in Appendix A.

Next, we briefly summarize some of the notation used in this paper: All $m \times m$ matrices $M \in \mathbb{C}^{m \times m}$ will be considered over the field of complex numbers $\mathbb{C}$. Moreover, $I_{m}$ denotes the identity matrix in $\mathbb{C}^{m \times m}$ for $m \in \mathbb{N}, M^{*}$ the adjoint (i.e., complex conjugate transpose), $M^{\top}$ the transpose of the matrix $M,\left[M_{1}, M_{2}\right]=$ $M_{1} M_{2}-M_{2} M_{1}$ denotes the standard commutator of two matrices $M_{j} \in \mathbb{C}^{m \times m}$, $j=1,2$.

$A C([a, b])$ (resp., $\left.A C_{\text {loc }}(c, d)\right)$ ) denotes the set of (locally) absolutely continuous functions on $[a, b]$ (resp., $(c, d))$. We also agree that $L^{2}((a, b))^{m}, m \in \mathbb{N}$, without explicit depiction of a measure always denotes $L^{2}((a, b) ; d x)^{m}$, with $d x$ representing the Lebesgue measure restricted to $(a, b),-\infty \leqslant a<b \leqslant \infty$, in particular,

$$
L^{2}((a, b))^{m}=\left\{U:(a, b) \rightarrow \mathbb{C}^{m} \mid \int_{a}^{b} d x\|U(x)\|_{\mathbb{C}^{m}}^{2}<\infty\right\}, \quad m \in \mathbb{N} .
$$

The identity operator in $L^{2}((a, b))^{m}$ will simply be denoted by $I$.

For ease of notation we will typically use the short cut $\left[x_{0}, \pm \infty\right)$ to denote the half-lines $\left[x_{0}, \infty\right)$ or $\left(-\infty, x_{0}\right]$ for some $x_{0} \in \mathbb{R}$.

Finally, let $T$ be a linear operator mapping (a subspace of) a Hilbert space into another, with $\operatorname{dom}(T), \operatorname{ran}(T)$, and $\operatorname{ker}(T)$ denoting the domain, range, and kernel (i.e., null space) of $T$. The closure of a closable operator $S$ is denoted by $\bar{S}$. The spectrum and resolvent set of a closed linear operator in a Hilbert space will be denoted by $\sigma(\cdot)$ and $\rho(\cdot)$, respectively.

\section{Weyl-Titchmarsh Matrices for Supersymmetric Dirac Operators}

In this preparatory section we briefly review the Weyl-Titchmarsh theory for Dirac-type operators $D$ in the special supersymmetric case. In particular, $D$ is constructed as a special case of the theory of singular Hamiltonian systems as pioneered by Hinton and Shaw [59]-63] (see also [57, [58]) and applied to Diractype operators in 16 .

Throughout this section we closely follow the treatment in [16] (simplified to the present supersymmetric Dirac-type operator) and hence are making the following assumptions.

Hypothesis 2.1. Suppose $\phi \in L_{\mathrm{loc}}^{1}(\mathbb{R})^{m \times m}, m \in \mathbb{N}$, and $\phi(\cdot)=\phi(\cdot)^{*}$ a.e. on $\mathbb{R}$.

Given Hypothesis 2.1 we introduce the maximally defined operators $A$ and $A^{+}$ in $L^{2}(\mathbb{R})^{m}$ by

$$
\begin{aligned}
& (A u)(x)=u^{\prime}(x)+\phi(x) u(x) \text { for a.e. } x \in \mathbb{R}, \\
& u \in \operatorname{dom}(A)=\left\{v \in L^{2}(\mathbb{R})^{m} \mid v \in A C_{\mathrm{loc}}(\mathbb{R}) ;\left(v^{\prime}+\phi v\right) \in L^{2}(\mathbb{R})^{m}\right\},
\end{aligned}
$$

and

$$
\begin{aligned}
& \left(A^{+} u\right)(x)=-u^{\prime}(x)+\phi(x) u(x) \text { for a.e. } x \in \mathbb{R} \\
& u \in \operatorname{dom}\left(A^{+}\right)=\left\{v \in L^{2}(\mathbb{R})^{m} \mid v \in A C_{\mathrm{loc}}(\mathbb{R}) ;\left(v^{\prime}-\phi v\right) \in L^{2}(\mathbb{R})^{m}\right\} .
\end{aligned}
$$


In addition, we consider the maximally defined Dirac-type operator $D$ in $L^{2}(\mathbb{R})^{2 m}$ by

$$
\begin{gathered}
(D U)(x)=\left(\left(\begin{array}{cc}
0 & A^{+} \\
A & 0
\end{array}\right)\left(\begin{array}{l}
u_{1} \\
u_{2}
\end{array}\right)\right)(x)=\left(\begin{array}{c}
\left(A^{+} u_{2}\right)(x) \\
\left(A u_{1}\right)(x)
\end{array}\right) \text { for a.e. } x \in \mathbb{R} \\
U=\left(\begin{array}{l}
u_{1} \\
u_{2}
\end{array}\right) \in \operatorname{dom}(D)=\operatorname{dom}(A) \oplus \operatorname{dom}\left(A^{+}\right) \\
=\left\{V=\left(\begin{array}{l}
v_{1} \\
v_{2}
\end{array}\right) \in L^{2}(\mathbb{R})^{2 m} \mid V \in A C_{\mathrm{loc}}(\mathbb{R})^{2 m} ; D V \in L^{2}(\mathbb{R})^{2 m}\right\} .
\end{gathered}
$$

The basic known result on $A, A^{+}$, and $D$ then reads as follows:

Theorem 2.2 ([16], [59], 61], 62]). Assume Hypothesis 2.1. Then $A$ and $A^{+}$are closed in $L^{2}(\mathbb{R})^{m}$ and

$$
A^{*}=A^{+}, \quad\left(A^{+}\right)^{*}=A .
$$

In addition, $D$ is self-adjoint in $L^{2}(\mathbb{R})^{2 m}$, that is, $D$ is of the form,

$$
D=\left(\begin{array}{cc}
0 & A^{*} \\
A & 0
\end{array}\right) \text {. }
$$

Proof. By [16, Lemma 2.15], the differential expression

$$
\mathcal{D}=\left(\begin{array}{cc}
0 & -I_{m}(d / d x)+\phi(x) \\
I_{m}(d / d x)+\phi(x) & 0
\end{array}\right) \text { for a.e. } x \in \mathbb{R},
$$

is in the limit point case at $\pm \infty$. (For a subsequent and more general result we refer to [96], see also [95] and [97] for such proofs under stronger hypotheses on $\phi)$. Combining this result with the Weyl-Titchmarsh theory developed for singular Hamiltonian systems by Hinton and Shaw in a series of papers [59, 61, 62, yields self-adjointness of the maximal operator associated to the differential expression $\mathcal{D}$. By (A.2), $A$ and $A^{+}$are hence necessarily closed, and consequently, adjoint to each other, proving (2.4) and (2.5).

Because of the special structure (2.5), $D$ is called a supersymmetric Dirac-type operator. For a discussion of its general properties we refer to Appendix $\mathrm{A}$.

Because of (2.4), we identify $A^{+}$and $A^{*}$ from this point on.

In order to discuss $m \times m$ Weyl-Titchmarsh matrices corresponding to $D$ on the half-lines $\left(-\infty, x_{0}\right]$ and $\left[x_{0}, \infty\right)$, we introduce boundary condition parameters $\alpha=\left(\alpha_{1} \alpha_{2}\right) \in \mathbb{C}^{m \times 2 m}$ satisfying the conditions

$$
\alpha \alpha^{*}=I_{m}, \quad \alpha J \alpha^{*}=0, \text { where } J=\left(\begin{array}{cc}
0 & -I_{m} \\
I_{m} & 0
\end{array}\right) .
$$

Explicitly, this reads

$$
\alpha_{1} \alpha_{1}^{*}+\alpha_{2} \alpha_{2}^{*}=I_{m}, \quad \alpha_{2} \alpha_{1}^{*}-\alpha_{1} \alpha_{2}^{*}=0 .
$$

In fact, one also has

$$
\alpha_{1}^{*} \alpha_{1}+\alpha_{2}^{*} \alpha_{2}=I_{m}, \quad \alpha_{2}^{*} \alpha_{1}-\alpha_{1}^{*} \alpha_{2}=0,
$$

as is clear from

$$
\left(\begin{array}{cc}
\alpha_{1} & \alpha_{2} \\
-\alpha_{2} & \alpha_{1}
\end{array}\right)\left(\begin{array}{cc}
\alpha_{1}^{*} & -\alpha_{2}^{*} \\
\alpha_{2}^{*} & \alpha_{1}^{*}
\end{array}\right)=I_{2 m}=\left(\begin{array}{cc}
\alpha_{1}^{*} & -\alpha_{2}^{*} \\
\alpha_{2}^{*} & \alpha_{1}^{*}
\end{array}\right)\left(\begin{array}{cc}
\alpha_{1} & \alpha_{2} \\
-\alpha_{2} & \alpha_{1}
\end{array}\right)
$$


since any left inverse matrix is also a right inverse, and vice versa. Moreover, from (2.9) one obtains

$$
\alpha^{*} \alpha J+J \alpha^{*} \alpha=J
$$

The particular choice where $\alpha$ equals

$$
\alpha_{0}=\left(I_{m} 0\right)
$$

will play a fundamental role later on.

Next, denote by $U_{ \pm}\left(\zeta, \cdot, x_{0}, \alpha\right)$ the $2 m \times m$ matrix-valued Weyl-Titchmarsh solutions associated with $\mathcal{D} U=\zeta U, \zeta \in \mathbb{C} \backslash \mathbb{R}$, defined by the property that the $m$ columns of $U_{ \pm}$span the deficiency spaces $N(\zeta, \pm \infty), \zeta \in \mathbb{C} \backslash \mathbb{R}$, given by

$$
\begin{gathered}
N(\zeta, \pm \infty)=\left\{V \in L^{2}\left(\left(x_{0}, \pm \infty\right)\right)^{2 m} \mid V \in A C\left(\left[x_{0}, x_{0} \pm R\right]\right)^{2 m} \text { for all } R>0\right. \\
\left.\mathcal{D} V=\zeta V \text { a.e. on }\left(x_{0}, \pm \infty\right)\right\}
\end{gathered}
$$

and normalized such that

$$
\begin{aligned}
U_{ \pm}\left(\zeta, x, x_{0}, \alpha\right) & =\left(\begin{array}{l}
u_{ \pm, 1}\left(\zeta, x, x_{0}, \alpha\right) \\
u_{ \pm, 2}\left(\zeta, x, x_{0}, \alpha\right)
\end{array}\right)=\Psi\left(\zeta, x, x_{0}, \alpha\right)\left(\begin{array}{c}
I_{m} \\
M_{ \pm}^{D}\left(\zeta, x_{0}, \alpha\right)
\end{array}\right) \\
& =\left(\begin{array}{cc}
\vartheta_{1}\left(\zeta, x, x_{0}, \alpha\right) & \varphi_{1}\left(\zeta, x, x_{0}, \alpha\right) \\
\vartheta_{2}\left(\zeta, x, x_{0}, \alpha\right) & \varphi_{2}\left(\zeta, x, x_{0}, \alpha\right)
\end{array}\right)\left(\begin{array}{c}
I_{m} \\
M_{ \pm}^{D}\left(\zeta, x_{0}, \alpha\right)
\end{array}\right) .
\end{aligned}
$$

Here $M_{ \pm}^{D}\left(\zeta, x_{0}, \alpha\right)$ represents an $m \times m$ matrix, and $\Psi\left(\zeta, x, x_{0}, \alpha\right), \vartheta_{j}\left(\zeta, x, x_{0}, \alpha\right)$, and $\varphi_{j}\left(\zeta, x, x_{0}, \alpha\right), j=1,2$, are defined as follows: $\Psi\left(\zeta, x, x_{0}, \alpha\right)$ satisfies $\mathcal{D} \Psi=\zeta \Psi$ a.e. on $\mathbb{R}$, normalized such that

$$
\Psi\left(\zeta, x_{0}, x_{0}, \alpha\right)=\left(\alpha^{*} J \alpha^{*}\right)=\left(\begin{array}{cc}
\alpha_{1}^{*} & -\alpha_{2}^{*} \\
\alpha_{2}^{*} & \alpha_{1}^{*}
\end{array}\right) .
$$

Partitioning $\Psi\left(\zeta, x, x_{0}, \alpha\right)$ as follows,

$$
\Psi\left(\zeta, x, x_{0}, \alpha\right)=\left(\begin{array}{ll}
\vartheta_{1}\left(\zeta, x, x_{0}, \alpha\right) & \varphi_{1}\left(\zeta, x, x_{0}, \alpha\right) \\
\vartheta_{2}\left(\zeta, x, x_{0}, \alpha\right) & \varphi_{2}\left(\zeta, x, x_{0}, \alpha\right)
\end{array}\right)
$$

defines $\vartheta_{j}\left(\zeta, x, x_{0}, \alpha\right)$ and $\varphi_{j}\left(\zeta, x, x_{0}, \alpha\right), j=1,2$, as $m \times m$ matrices, entire with respect to $\zeta \in \mathbb{C}$, and normalized according to (2.15).

The matrices $M_{ \pm}^{D}\left(\zeta, x_{0}, \alpha\right)$ represent the sought after half-line Weyl-Titchmarsh matrices associated with the Dirac-type operator $D$, whose basic properties can be summarized as follows:

Theorem 2.3 ([5], 6], [15], [16], 47], 59], 60], 63], [93]).

Suppose Hypothesis 2.1 , let $\zeta \in \mathbb{C} \backslash \mathbb{R}, x_{0} \in \mathbb{R}$, and denote by $\alpha, \gamma \in \mathbb{C}^{m \times 2 m}$ matrices satisfying (2.7). Then the following hold:

( $i) \pm M_{ \pm}^{D}\left(\cdot, x_{0}, \alpha\right)$ is an $m \times m$ matrix-valued Nevanlinna-Herglotz function of maximal rank $m$. In particular,

$$
\begin{aligned}
& \operatorname{Im}\left( \pm M_{ \pm}^{D}\left(\zeta, x_{0}, \alpha\right)\right) \geqslant 0, \quad \zeta \in \mathbb{C}_{+}, \\
& M_{ \pm}^{D}\left(\bar{\zeta}, x_{0}, \alpha\right)=M_{ \pm}^{D}\left(\zeta, x_{0}, \alpha\right)^{*}, \\
& \operatorname{rank}\left(M_{ \pm}^{D}\left(\zeta, x_{0}, \alpha\right)\right)=m, \\
& \lim _{\varepsilon \downarrow 0} M_{ \pm}^{D}\left(\nu+i \varepsilon, x_{0}, \alpha\right) \text { exists for a.e. } \nu \in \mathbb{R}, \\
& M_{ \pm}^{D}\left(\zeta, x_{0}, \alpha\right)=\left[-\alpha J \gamma^{*}+\alpha \gamma^{*} M_{ \pm}^{D}\left(\zeta, x_{0}, \gamma\right)\right]\left[\alpha \gamma^{*}+\alpha J \gamma^{*} M_{ \pm}^{D}\left(\zeta, x_{0}, \gamma\right)\right]^{-1} .
\end{aligned}
$$


Local singularities of $\pm M_{ \pm}^{D}\left(\cdot, x_{0}, \alpha\right)$ and $\mp M_{ \pm}^{D}\left(\cdot, x_{0}, \alpha\right)^{-1}$ are necessarily real and at most of first order in the sense that

$$
\mp \lim _{\epsilon \downarrow 0}\left(i \epsilon M_{ \pm}^{D}\left(\nu+i \epsilon, x_{0}, \alpha\right)\right) \geqslant 0, \quad \pm \lim _{\epsilon \downarrow 0}\left(i \epsilon M_{ \pm}^{D}\left(\nu+i \epsilon, x_{0}, \alpha\right)^{-1}\right) \geqslant 0, \quad \nu \in \mathbb{R} .
$$

(ii) $\pm M_{ \pm}^{D}\left(\cdot, x_{0}, \alpha\right)$ admits the representation

$$
\pm M_{ \pm}^{D}\left(\zeta, x_{0}, \alpha\right)=F_{ \pm}\left(x_{0}, \alpha\right)+\int_{\mathbb{R}} d \Omega_{ \pm}^{D}\left(\nu, x_{0}, \alpha\right)\left[(\nu-\zeta)^{-1}-\nu\left(1+\nu^{2}\right)^{-1}\right]
$$

where

$$
F_{ \pm}\left(x_{0}, \alpha\right)=F_{ \pm}\left(x_{0}, \alpha\right)^{*}, \quad \int_{\mathbb{R}}\left\|d \Omega_{ \pm}^{D}\left(\nu, x_{0}, \alpha\right)\right\|_{\mathbb{C}^{m \times m}}\left(1+\nu^{2}\right)^{-1}<\infty .
$$

Moreover,

$$
\Omega_{ \pm}^{D}\left((\mu, \nu], x_{0}, \alpha\right)=\lim _{\delta \downarrow 0} \lim _{\varepsilon \downarrow 0} \frac{1}{\pi} \int_{\mu+\delta}^{\nu+\delta} d \nu^{\prime} \operatorname{Im}\left( \pm M_{ \pm}^{D}\left(\nu^{\prime}+i \varepsilon, x_{0}, \alpha\right)\right) .
$$

(iii) $\operatorname{Im}\left(M_{ \pm}^{D}\left(\cdot, x_{0}, \alpha\right)\right)$ satisfies

$$
\begin{aligned}
& \operatorname{Im}\left(M_{ \pm}^{D}\left(\zeta, x_{0}, \alpha\right)\right)= \operatorname{Im}(\zeta) \int_{x_{0}}^{ \pm \infty} d x U_{ \pm}\left(\zeta, x, x_{0}, \alpha\right)^{*} U_{ \pm}\left(\zeta, x, x_{0}, \alpha\right) \\
&=\operatorname{Im}(\zeta) \int_{x_{0}}^{ \pm \infty} d x\left[\begin{array}{r}
u_{ \pm, 1}\left(\zeta, x, x_{0}, \alpha\right)^{*} u_{ \pm, 1}\left(\zeta, x, x_{0}, \alpha\right) \\
\left.\quad+u_{ \pm, 2}\left(\zeta, x, x_{0}, \alpha\right)^{*} u_{ \pm, 2}\left(\zeta, x, x_{0}, \alpha\right)\right]
\end{array}\right.
\end{aligned}
$$

For completeness we also recall that the $2 m \times 2 m$ Green's matrix (i.e., the integral kernel of the resolvent) of $D$ is given in terms of $U_{ \pm}$and $M_{ \pm}$by

$$
\begin{array}{r}
G^{D}\left(\zeta, x, x^{\prime}\right)=(D-\zeta I)^{-1}\left(x, x^{\prime}\right) \\
=U_{\mp}\left(\zeta, x, x_{0}, \alpha\right)\left[M_{-}^{D}\left(\zeta, x_{0}, \alpha\right)-M_{+}^{D}\left(\zeta, x_{0}, \alpha\right)\right]^{-1} U_{ \pm}\left(\bar{\zeta}, x^{\prime}, x_{0}, \alpha\right)^{*}, \\
x \lessgtr x^{\prime}, x, x^{\prime} \in \mathbb{R}, \zeta \in \mathbb{C} \backslash \mathbb{R} .
\end{array}
$$

Of course, $G^{D}\left(\zeta, x, x^{\prime}\right)$ is independent of the choice of reference point $x_{0} \in \mathbb{R}$, and independent of the boundary condition parameter $\alpha$ satisfying (2.7) used in $M_{ \pm}^{D}\left(\zeta, x_{0}, \alpha\right)$ and $U_{ \pm}\left(\zeta, \cdot, x_{0}, \alpha\right)$. One also notes that (2.27) extends as usual to all $\zeta \in \rho(D)$. In the particular case $\alpha_{0}=\left(I_{m} 0\right)$ one obtains

$$
U_{ \pm}\left(\zeta, x_{0}, x_{0}, \alpha_{0}\right)=\left(\begin{array}{l}
u_{ \pm, 1}\left(\zeta, x_{0}, x_{0}, \alpha_{0}\right) \\
u_{ \pm, 2}\left(\zeta, x_{0}, x_{0}, \alpha_{0}\right)
\end{array}\right)=\left(\begin{array}{c}
I_{m} \\
M_{ \pm}^{D}\left(\zeta, x_{0}, \alpha_{0}\right)
\end{array}\right)
$$

The self-adjoint half-line Dirac operators $D_{ \pm}(\alpha)$ in $L^{2}\left(\left[x_{0}, \pm \infty\right)\right)^{2 m}$ associated with a self-adjoint boundary condition at $x_{0}$ indexed by $\alpha \in \mathbb{C}^{m \times 2 m}$ satisfying (2.7), are of the form

$$
\begin{aligned}
\left(D_{ \pm}(\alpha) U\right)(x)=(\mathcal{D} U)(x) \text { for a.e. } x \in\left[x_{0}, \pm \infty\right), & \\
U \in \operatorname{dom}\left(D_{ \pm}(\alpha)\right)= & \left\{V \in L^{2}\left(\left[x_{0}, \pm \infty\right)\right)^{2 m} \mid V \in A C\left(\left[x_{0}, x_{0} \pm R\right]\right)^{2 m}\right. \\
& \left.\quad \text { for all } R>0 ; \alpha \phi\left(x_{0}\right)=0 ; \mathcal{D} V \in L^{2}\left(\left[x_{0}, \pm \infty\right)\right)^{2 m}\right\} .
\end{aligned}
$$

The $m \times m$ matrix-valued spectral function of $D_{ \pm}(\alpha)$ then generates the measure $\Omega_{ \pm}^{D}\left(\cdot, x_{0}, \alpha\right)$ in (2.23). 
We conclude this section with a brief description of the full-line $2 m \times 2 m$ WeylTitchmarsh matrix $\mathbf{M}^{D}\left(\zeta, x_{0}, \alpha\right)$ associated with $D$ as described in [59] 63$]$ :

$$
\begin{aligned}
\mathbf{M}^{D}\left(\zeta, x_{0}, \alpha\right)= & \left(\mathbf{M}_{j, j^{\prime}}^{D}\left(\zeta, x_{0}, \alpha\right)\right)_{j, j^{\prime}=1,2}, \quad \zeta \in \mathbb{C} \backslash \mathbb{R} \\
\mathbf{M}_{0,0}^{D}\left(\zeta, x_{0}, \alpha\right)= & {\left[M_{-}^{D}\left(\zeta, x_{0}, \alpha\right)-M_{+}^{D}\left(\zeta, x_{0}, \alpha\right)\right]^{-1} } \\
\mathbf{M}_{0,1}^{D}\left(\zeta, x_{0}, \alpha\right)= & 2^{-1}\left[M_{-}^{D}\left(\zeta, x_{0}, \alpha\right)-M_{+}^{D}\left(\zeta, x_{0}, \alpha\right)\right]^{-1} \\
& \times\left[M_{-}^{D}\left(\zeta, x_{0}, \alpha\right)+M_{+}^{D}\left(\zeta, x_{0}, \alpha\right)\right] \\
\mathbf{M}_{1,0}^{D}\left(\zeta, x_{0}, \alpha\right)= & 2^{-1}\left[M_{-}^{D}\left(\zeta, x_{0}, \alpha\right)+M_{+}^{D}\left(\zeta, x_{0}, \alpha\right)\right] \\
& \times\left[M_{-}^{D}\left(\zeta, x_{0}, \alpha\right)-M_{+}^{D}\left(\zeta, x_{0}, \alpha\right)\right]^{-1} \\
\mathbf{M}_{1,1}^{D}\left(\zeta, x_{0}, \alpha\right)= & M_{ \pm}^{D}\left(\zeta, x_{0}, \alpha\right)\left[M_{-}^{D}\left(\zeta, x_{0}, \alpha\right)-M_{+}^{D}\left(\zeta, x_{0}, \alpha\right)\right]^{-1} M_{\mp}^{D}\left(\zeta, x_{0}, \alpha\right)
\end{aligned}
$$

The basic results on $\mathbf{M}^{D}\left(\cdot, x_{0}, \alpha\right)$ then read as follows.

Theorem 2.4 (47, [59, 60, 63, 63]). Assume Hypothesis 2.1 and suppose that $\zeta \in \mathbb{C} \backslash \mathbb{R}, x_{0} \in \mathbb{R}$, and that $\alpha \in \mathbb{C}^{m \times 2 m}$ satisfies (2.7). Then the following hold:

(i) $\mathbf{M}^{D}\left(\cdot, x_{0}, \alpha\right)$ is a matrix-valued Nevanlinna-Herglotz function of maximal rank $2 m$ with representation

$$
\mathbf{M}^{D}\left(\zeta, x_{0}, \alpha\right)=\mathbf{F}\left(x_{0}, \alpha\right)+\int_{\mathbb{R}} d \boldsymbol{\Omega}^{D}\left(\nu, x_{0}, \alpha\right)\left[(\nu-\zeta)^{-1}-\nu\left(1+\nu^{2}\right)^{-1}\right],
$$

where

$$
\mathbf{F}\left(x_{0}, \alpha\right)=\mathbf{F}\left(x_{0}, \alpha\right)^{*}, \quad \int_{\mathbb{R}}\left\|d \boldsymbol{\Omega}^{D}\left(\nu, x_{0}, \alpha\right)\right\|_{\mathbb{C}^{2 m \times 2 m}}\left(1+\nu^{2}\right)^{-1}<\infty .
$$

Moreover,

$$
\boldsymbol{\Omega}^{D}\left((\mu, \nu], x_{0}, \alpha\right)=\lim _{\delta \downarrow 0} \lim _{\varepsilon \downarrow 0} \frac{1}{\pi} \int_{\mu+\delta}^{\nu+\delta} d \nu^{\prime} \operatorname{Im}\left(\mathbf{M}^{D}\left(\nu^{\prime}+i \varepsilon, x_{0}, \alpha\right)\right) .
$$

(ii) $\zeta \in \rho(D)$ if and only if $\mathbf{M}^{D}\left(\zeta, x_{0}, \alpha\right)$ is holomorphic near $\zeta$.

Finally, observe that supersymmetry implies various symmetries for the associated quantities.

Lemma 2.5. Assume Hypothesis 2.1. The operators $D_{ \pm}\left(\alpha_{0}\right)$ are supersymmetric and satisfy $\mathfrak{S}_{3} D_{ \pm}\left(\alpha_{0}\right) \mathfrak{S}_{3}=-D_{ \pm}\left(\alpha_{0}\right)$, where

$$
\mathfrak{S}_{3}=\left(\begin{array}{cc}
I_{m} & 0 \\
0 & -I_{m}
\end{array}\right) \text {. }
$$

Moreover,

$$
\begin{aligned}
& \mathfrak{S}_{3}\left(\varphi_{1}\left(\zeta, x, x_{0}, \alpha_{0}\right) \varphi_{2}\left(\zeta, x, x_{0}, \alpha_{0}\right)\right)^{\top}=-\left(\varphi_{1}\left(-\zeta, x, x_{0}, \alpha_{0}\right) \quad \varphi_{2}\left(-\zeta, x, x_{0}, \alpha_{0}\right)\right)^{\top}, \\
& \mathfrak{S}_{3}\left(\vartheta_{1}\left(\zeta, x, x_{0}, \alpha_{0}\right) \quad \vartheta_{2}\left(\zeta, x, x_{0}, \alpha_{0}\right)\right)^{\top}=\left(\vartheta_{1}\left(-\zeta, x, x_{0}, \alpha_{0}\right) \quad \vartheta_{2}\left(-\zeta, x, x_{0}, \alpha_{0}\right)\right)^{\top} \text {, } \\
& \mathfrak{S}_{3} U_{ \pm}\left(\zeta, x, x_{0}, \alpha_{0}\right)=U_{ \pm}\left(-\zeta, x, x_{0}, \alpha_{0}\right) \text {, } \\
& M_{ \pm}^{D}\left(\zeta, x_{0}, \alpha_{0}\right)=-M_{ \pm}^{D}\left(-\zeta, x_{0}, \alpha_{0}\right), \\
& d \Omega_{ \pm}^{D}\left(\nu, x_{0}, \alpha_{0}\right)=d \Omega_{ \pm}^{D}\left(-\nu, x_{0}, \alpha_{0}\right) .
\end{aligned}
$$

Similarly, $D$ is supersymmetric, $\mathfrak{S}_{3} D \mathfrak{S}_{3}=-D$, and

$$
\begin{aligned}
& \mathbf{M}^{D}\left(\zeta, x_{0}, \alpha_{0}\right)=-\mathfrak{S}_{3} \mathbf{M}^{D}\left(-\zeta, x_{0}, \alpha_{0}\right) \mathfrak{S}_{3}, \\
& d \boldsymbol{\Omega}^{D}\left(\nu, x_{0}, \alpha_{0}\right)=\mathfrak{S}_{3} d \boldsymbol{\Omega}^{D}\left(-\nu, x_{0}, \alpha_{0}\right) \mathfrak{S}_{3} .
\end{aligned}
$$


Proof. Clearly $D_{ \pm}\left(\alpha_{0}\right)$ are of the form (A.2) in this case and hence they are supersymmetric. Moreover, the symmetries for the solutions follow since both sides satisfy the same differential equation and the same initial conditions, respectively normalizations. The claims for $D$ are immediate from the ones for $D_{ \pm}\left(\alpha_{0}\right)$.

\section{Supersymmetry and the connection Between the Weyl-Titchmarsh} Matrices for Dirac and Generalized Schrödinger-Type Operators

In our principal section we provide the connection with the matrix-valued WeylTitchmarsh functions of the supersymmetric Dirac-type operator $D$ described in Section 2 and two naturally associated generalized Schrödinger-type operators $H_{j}$, $j=1,2$, given by

$$
D^{2}=\left(\begin{array}{cc}
A^{*} A & 0 \\
0 & A A^{*}
\end{array}\right)=H_{1} \oplus H_{2} \text { in } L^{2}(\mathbb{R})^{2 m} \simeq L^{2}(\mathbb{R})^{m} \oplus L^{2}(\mathbb{R})^{m},
$$

in particular, we denote

$$
H_{1}=A^{*} A, \quad H_{2}=A A^{*},
$$

with $A$ and $A^{*}$ given by (2.1) and (2.2), respectively (cf. Theorem 2.2).

While $D$ contains the locally integrable $m \times m$ matrix-valued coefficient $\phi$, the associated generalized Schrödinger operators $H_{j}, j=1,2$, will exhibit distributional potentials and hence are outside the standard Weyl-Titchmarsh theory for SturmLiouville operators with locally integrable $m \times m$ matrix-valued potentials. Our supersymmetric approach will enable us to make the transition from the usual $L_{\text {loc }}^{1}$-potentials in Schrödinger operators to distributional $H_{\text {loc }}^{-1}$-potentials (and more general situations) in an effortless manner, thereby underscoring the power of these supersymmetric arguments.

To describe $H_{j}, j=1,2$, in $L^{2}(\mathbb{R})$ in detail, we first introduce the following two kinds of quasi-derivatives,

$$
\begin{aligned}
& u^{[1,1]}(x)=(A u)(x)=u^{\prime}(x)+\phi(x) u(x) \text { for a.e. } x \in \mathbb{R}, \quad u \in \operatorname{dom}(A), \\
& v^{[1,2]}(x)=-\left(A^{*} v\right)(x)=v^{\prime}(x)-\phi(x) v(x) \text { for a.e. } x \in \mathbb{R}, \quad v \in \operatorname{dom}\left(A^{*}\right) .
\end{aligned}
$$

Thus, one infers,

$$
\begin{gathered}
\left(H_{1} u\right)(x)=\left(A^{*} A u\right)(x)=\left(\tau_{1} u\right)(x)=-\left(u^{[1,1]}\right)^{\prime}(x)+\phi(x) u^{[1,1]}(x) \text { for a.e. } x \in \mathbb{R}, \\
u \in \operatorname{dom}\left(H_{1}\right)=\left\{v \in L^{2}(\mathbb{R})^{m} \mid v, v^{[1,1]} \in A C_{\mathrm{loc}}(\mathbb{R})^{m}\right. \\
\left.\left[\left(v^{[1,1]}\right)^{\prime}+\phi v^{[1,1]}\right] \in L^{2}(\mathbb{R})^{m}\right\}
\end{gathered}
$$

and

$$
\begin{gathered}
\left(H_{2} u\right)(x)=\left(A A^{*} u\right)(x)=\left(\tau_{2} u\right)(x)=-\left(u^{[1,2]}\right)^{\prime}(x)-\phi(x) u^{[1,2]}(x) \text { for a.e. } x \in \mathbb{R}, \\
u \in \operatorname{dom}\left(H_{2}\right)=\left\{v \in L^{2}(\mathbb{R})^{m} \mid v, v^{[1,2]} \in A C_{\mathrm{loc}}(\mathbb{R})^{m}\right. \\
\left.\left[\left(v^{[1,2]}\right)^{\prime}+\phi v^{[1,2]}\right] \in L^{2}(\mathbb{R})^{m}\right\}
\end{gathered}
$$

Formally, $\tau_{j}, j=1,2$, are of the form

$$
\tau_{j}=-I_{m} \frac{d^{2}}{d x^{2}}+V_{j}(x), \quad V_{j}(x)=\phi(x)^{2}+(-1)^{j} \phi^{\prime}(x), \quad j=1,2,
$$

but one notices that, in general, neither $\phi^{2}$ is locally integrable (unless one makes the stronger assumption $\phi \in L_{\text {loc }}^{2}(\mathbb{R})^{m \times m}$ ), nor is $\phi^{\prime}$ a function (unless one assumes in addition that $\left.\phi \in A C_{\text {loc }}(\mathbb{R})^{m \times m}\right)$. 
By inspection, the second-order initial value problems,

$$
\begin{aligned}
& \left(\left(\tau_{j}-z\right) f\right)(x)=g(x) \text { for a.e. } x \in \mathbb{R}, \quad f, f^{[1, j]} \in A C_{\mathrm{loc}}(\mathbb{R})^{m}, g \in L_{\mathrm{loc}}^{1}(\mathbb{R})^{m}, \\
& f\left(x_{0}\right)=c_{0}, f^{[1, j]}\left(x_{0}\right)=d_{0}, j=1,2,
\end{aligned}
$$

for some $x_{0} \in \mathbb{R}, c_{0}, d_{0} \in \mathbb{C}$, are equivalent to the first-order initial value problems

$$
\begin{aligned}
& \left(\begin{array}{c}
f(x) \\
f^{[1, j]}(x)
\end{array}\right)^{\prime}=\left(\begin{array}{cc}
(-1)^{j} \phi(x) & 1 \\
-z & (-1)^{j+1} \phi(x)
\end{array}\right)\left(\begin{array}{c}
f(x) \\
f^{[1, j]}(x)
\end{array}\right)-\left(\begin{array}{c}
0 \\
g(x)
\end{array}\right) \text { for a.e. } x \in \mathbb{R} \\
& \left(\begin{array}{c}
f\left(x_{0}\right) \\
f^{[1, j]}\left(x_{0}\right)
\end{array}\right)=\left(\begin{array}{c}
c_{0} \\
d_{0}
\end{array}\right), \quad j=1,2,
\end{aligned}
$$

respectively. Since by Hypothesis $2.1, \phi \in L_{\text {loc }}^{1}(\mathbb{R})^{m \times m}$, the initial value problems in (3.9) (and hence those in (3.8)) are uniquely solvable by [111, Theorem 16.1] (see also [33, Theorem 10.1] and [111, Theorem 16.2]).

Next, suppose that for some $1 \leqslant p \leqslant m, U=\left(u_{1} u_{2}\right)^{\top}$ is a distributional $2 m \times p$ solution of $D U=\zeta U$, that is,

$$
\begin{aligned}
& u_{j} \in A C_{\mathrm{loc}}(\mathbb{R})^{m \times p}, j=1,2, \\
& u_{1}^{[1,1]}=A u_{1} \in L_{\mathrm{loc}}^{1}(\mathbb{R})^{m \times p}, \quad u_{2}^{[1,2]}=-A^{*} u_{2} \in L_{\mathrm{loc}}^{1}(\mathbb{R})^{m \times p} .
\end{aligned}
$$

Then, if $\zeta \neq 0$, the supersymmetric structure of $D$ in (2.5) actually implies that also

$$
\begin{aligned}
& u_{1}^{[1,1]}=A u_{1}=\zeta u_{2} \in A C_{\mathrm{loc}}(\mathbb{R})^{m \times p}, \\
& u_{2}^{[1,2]}=-A^{*} u_{2}=-\zeta u_{1} \in A C_{\mathrm{loc}}(\mathbb{R})^{m \times p},
\end{aligned}
$$

and hence that $u_{j}$ are actually distributional $m \times p$ solutions of $H_{j} u=\zeta^{2} u, j=1,2$, that is,

$$
\begin{aligned}
& u_{j}, u_{j}^{[1, j]} \in A C_{\mathrm{loc}}(\mathbb{R})^{m \times p}, \quad\left(u_{j}^{[1, j]}\right)^{\prime} \in L_{\mathrm{loc}}^{1}(\mathbb{R})^{m \times p}, \\
& \tau_{j} u_{j}=-\left(u_{j}^{[1, j]}\right)^{\prime}+(-1)^{j+1} \phi u_{j}^{[1, j]}=\zeta^{2} u_{j}, \quad j=1,2 .
\end{aligned}
$$

Thus, applying the $L^{2}$-property (2.26) and (3.10)-(3.13) to the Weyl-Titchmarsh solutions $U_{ \pm}\left(\zeta, \cdot, x_{0}, \alpha\right)$ associated with the Dirac-type operator $D$, then shows that $u_{ \pm, j}\left(\zeta, \cdot, x_{0}, \alpha\right)$ are Weyl-Titchmarsh solutions associated with $H_{j}, j=1,2$, replacing the complex energy parameter $\zeta$ by $z=\zeta^{2}$. Moreover, introducing the following fundamental system $s_{j}\left(z, \cdot, x_{0}\right), c_{j}\left(z, \cdot, x_{0}\right), j=1,2$, of $m \times m$ matrix solutions of $\tau_{j} u=z u, z \in \mathbb{C}, j=1,2$, normalized for arbitrary $z \in \mathbb{C}$ by

$$
\begin{array}{ll}
s_{j}\left(z, x_{0}, x_{0}\right)=0, & s_{j}^{[1, j]}\left(z, x_{0}, x_{0}\right)=I_{m}, \\
c_{j}\left(z, x_{0}, x_{0}\right)=I_{m}, & c_{j}^{[1, j]}\left(z, x_{0}, x_{0}\right)=0, \quad j=1,2,
\end{array}
$$

one observes as usual that for fixed $x, x_{0} \in \mathbb{R}, s_{j}\left(\cdot, x, x_{0}\right), c_{j}\left(\cdot, x, x_{0}\right)$ are entire. The connection with the solutions $\varphi_{j}$ and $\vartheta_{j}, j=1,2$, of $D$ is given by

$$
\begin{array}{ll}
s_{1}\left(z, x, x_{0}\right)=\zeta^{-1} \varphi_{1}\left(\zeta, x, x_{0}, \alpha_{0}\right), & c_{1}\left(z, x, x_{0}\right)=\vartheta_{1}\left(\zeta, x, x_{0}, \alpha_{0}\right), \\
s_{2}\left(z, x, x_{0}\right)=\zeta^{-1} \vartheta_{2}\left(\zeta, x, x_{0}, \alpha_{0}\right), & c_{2}\left(z, x, x_{0}\right)=\varphi_{2}\left(\zeta, x, x_{0}, \alpha_{0}\right), \quad z=\zeta^{2} .
\end{array}
$$

In addition, introducing the Weyl-Titchmarsh solutions $\psi_{ \pm, j}\left(z, \cdot, x_{0}\right)$ for $H_{j}, j=$ 1,2 , via

$$
\psi_{ \pm, 1}\left(z, \cdot, x_{0}\right)=u_{ \pm, 1}\left(\zeta, \cdot, x_{0}, \alpha_{0}\right)
$$




$$
\begin{array}{r}
\psi_{ \pm, 2}\left(z, \cdot, x_{0}\right)=u_{ \pm, 2}\left(\zeta, \cdot, x_{0}, \alpha_{0}\right) M_{ \pm}^{D}\left(\zeta, x_{0}, \alpha_{0}\right)^{-1}, \\
z=\zeta^{2}, \zeta \in \mathbb{C} \backslash \mathbb{R}, j=1,2,
\end{array}
$$

(the right-hand sides being independent of the choice of branch for $\zeta$ ) and the generalized Dirichlet-type $m \times m$ matrix-valued Weyl-Titchmarsh functions $\widehat{M}_{ \pm, 0, j}\left(\cdot, x_{0}\right)$ of $H_{j}$,

$$
\begin{array}{r}
\widehat{M}_{ \pm, 0,1}\left(z, x_{0}\right)=\zeta M_{ \pm}^{D}\left(\zeta, x_{0}, \alpha_{0}\right), \\
\widehat{M}_{ \pm, 0,2}\left(z, x_{0}\right)=-\zeta M_{ \pm}^{D}\left(\zeta, x_{0}, \alpha_{0}\right)^{-1}, \\
z=\zeta^{2}, \zeta \in \mathbb{C} \backslash \mathbb{R},
\end{array}
$$

one infers from (2.28) that

$$
\psi_{ \pm, j}\left(z, \cdot, x_{0}\right)=c_{j}\left(z, \cdot, x_{0}\right)+s_{j}\left(z, \cdot, x_{0}\right) \widehat{M}_{ \pm, 0, j}\left(z, x_{0}\right), \quad z \in \mathbb{C} \backslash[0, \infty), j=1,2 .
$$

Indeed, (3.22) follows from combining (2.28), (3.11), and (3.12) (for $p=m$ ), which in turn imply

$$
\begin{aligned}
\psi_{ \pm, j}\left(z, x_{0}, x_{0}\right) & =I_{m}, \\
\psi_{ \pm, j}^{[1, j]}\left(z, x_{0}, x_{0}\right) & =\widehat{M}_{ \pm, 0, j}\left(z, x_{0}\right), \quad j=1,2
\end{aligned}
$$

and the unique solvability of the initial value problems in (3.8). We summarize this discussion in the following result:

Theorem 3.1. Assume Hypothesis 2.1 and let $\alpha_{0}=\left(I_{m} 0\right)$. Suppose that the corresponding Weyl-Titchmarsh solutions of $D$ are denoted by $U_{ \pm}\left(\zeta, \cdot, x_{0}, \alpha_{0}\right)=$ $\left(u_{ \pm, 1}\left(\zeta, \cdot, x_{0}, \alpha_{0}\right) u_{ \pm, 2}\left(\zeta, \cdot, x_{0}, \alpha_{0}\right)\right)^{\top}$ and the corresponding $m \times m$ matrix-valued half-line Weyl-Titchmarsh function of $D$ is given by $M_{ \pm}^{D}\left(\cdot, x_{0}, \alpha_{0}\right)$. Then the $m \times m$ matrix-valued Weyl-Titchmarsh solutions of $H_{j}$, denoted by $\psi_{ \pm, j}\left(z, \cdot, x_{0}\right), j=1,2$, are given by (3.18) and (3.19), and the $m \times m$ matrix-valued generalized Dirichlettype Weyl-Titchmarsh functions $\widehat{M}_{ \pm, 0, j}\left(\cdot, x_{0}\right)$ of $H_{j}, j=1,2$, are given by (3.20) and (3.21). In particular,

$$
\widehat{M}_{ \pm, 0,1}\left(z, x_{0}\right)=\zeta M_{ \pm}^{D}\left(\zeta, x_{0}, \alpha_{0}\right)=-z \widehat{M}_{ \pm, 0,2}\left(z, x_{0}\right)^{-1}, \quad z=\zeta^{2}, \zeta \in \mathbb{C} \backslash \mathbb{R}
$$

A version of the equality $\widehat{M}_{ \pm, 0,1}\left(z, x_{0}\right)=-z \widehat{M}_{ \pm, 0,2}\left(z, x_{0}\right)^{-1}$, in the special scalar case $m=1$, and under the stronger hypothesis $\phi \in A C_{\text {loc }}(\mathbb{R})$, first appeared in 42 , eq. (5.71)], and was quoted again in [46, eq. (A.25)].

The subscript " 0 " in $\widehat{M}_{ \pm, 0, j}\left(z, x_{0}\right), j=1,2$, indicates that these generalized Weyl-Titchmarsh matrices correspond to a Dirichlet boundary condition at the reference point $x_{0}$ in the corresponding generalized half-line Schrödinger operators $H_{ \pm, 0, j}, j=1,2$, in $L^{2}\left(\left[x_{0}, \pm \infty\right)\right)^{m}$ defined by

$$
\begin{aligned}
& \left(H_{ \pm, 0, j} u\right)(x) \\
& \quad=\left(\tau_{j} u\right)(x)=-\left(u^{[1, j]}\right)^{\prime}(x)+(-1)^{j+1} \phi(x) u^{[1, j]}(x) \text { for a.e. } x \in\left[x_{0}, \pm \infty\right), \\
& u \in \operatorname{dom}\left(H_{ \pm, 0, j}\right) \\
& =\left\{v \in L^{2}\left(\left[x_{0}, \pm \infty\right)\right)^{m} \mid v, v^{[1, j]} \in A C\left(\left[x_{0}, x_{0} \pm R\right]\right)^{m} \text { for all } R>0 ;\right. \\
& \left.v\left(x_{0}\right)=0 ;\left[\left(v^{[1, j]}\right)^{\prime}+(-1)^{j} \phi v^{[1, j]}\right] \in L^{2}\left(\left[x_{0}, \pm \infty\right)\right)^{m}\right\} \\
& j=1,2 .
\end{aligned}
$$


The corresponding Green's function of $H_{ \pm, 0, j}$ is then of the familiar form

$$
\begin{aligned}
G_{+, 0, j}\left(z, x, x^{\prime}\right)= & \left(H_{+, 0, j}-z I\right)^{-1}\left(x, x^{\prime}\right) \\
= & \begin{cases}s_{j}\left(z, x, x_{0}\right) \psi_{+, j}\left(\bar{z}, x^{\prime}, x_{0}\right)^{*}, & x \leqslant x^{\prime}, \\
\psi_{+, j}\left(z, x, x_{0}\right) s_{j}\left(\bar{z}, x^{\prime}, x_{0}\right)^{*}, & x^{\prime} \leqslant x,\end{cases} \\
& x, x^{\prime} \in\left[x_{0}, \infty\right), z \in \mathbb{C} \backslash[0, \infty), j=1,2,
\end{aligned}
$$

and

$$
\begin{aligned}
G_{-, 0, j}\left(z, x, x^{\prime}\right)= & \left(H_{-, 0, j}-z I\right)^{-1}\left(x, x^{\prime}\right) \\
= & - \begin{cases}s_{j}\left(z, x, x_{0}\right) \psi_{-, j}\left(\bar{z}, x^{\prime}, x_{0}\right)^{*}, & x^{\prime} \leqslant x, \\
\psi_{-, j}\left(z, x, x_{0}\right) s_{j}\left(\bar{z}, x^{\prime}, x_{0}\right)^{*}, & x \leqslant x^{\prime},\end{cases} \\
& x, x^{\prime} \in\left(-\infty, x_{0}\right], z \in \mathbb{C} \backslash[0, \infty), j=1,2 .
\end{aligned}
$$

Similarly, the diagonal terms in (A.33) together with (2.18), (2.27) and (3.18)-(3.22) yield the Green's function for $H_{j}$,

$$
\begin{aligned}
& G_{j}\left(z, x, x^{\prime}\right)=\left(H_{j}-z I\right)^{-1}\left(x, x^{\prime}\right) \\
& =\psi_{\mp, j}\left(z, x, x_{0}\right)\left[\widehat{M}_{-, 0, j}\left(z, x_{0}\right)-\widehat{M}_{+, 0, j}\left(z, x_{0}\right)\right]^{-1} \psi_{ \pm, j}\left(\bar{z}, x^{\prime}, x_{0}\right)^{*}, \\
& x \lesseqgtr x^{\prime}, x, x^{\prime} \in \mathbb{R}, z \in \mathbb{C} \backslash[0, \infty), j=1,2 .
\end{aligned}
$$

One can show that

$$
\begin{aligned}
\widehat{M}_{-, 0, j}\left(z, x_{0}\right)-\widehat{M}_{+, 0, j}\left(z, x_{0}\right) & =W\left(\psi_{+, j}\left(\bar{z}, \cdot, x_{0}\right)^{*}, \psi_{-, j}\left(z, \cdot, x_{0}\right)\right) \\
& =-W\left(\psi_{-, j}\left(\bar{z}, \cdot, x_{0}\right)^{*}, \psi_{+, j}\left(z, \cdot, x_{0}\right)\right), \quad j=1,2,
\end{aligned}
$$

where $W(\cdot, \cdot), j=1,2$, denote the Wronskians of matrix-valued functions $F, G \in$ $A C_{\text {loc }}(\mathbb{R})^{m \times m}$ defined by

$$
\begin{aligned}
W(F, G)(x) & =F(x) G^{\prime}(x)-F^{\prime}(x) G(x) \\
& =F(x) G^{[1, j]}(x)-F^{[1, j]}(x) G(x)+(-1)^{j}[F(x), \phi(x)] G(x), \\
& \text { for a.e. } x \in \mathbb{R}, \quad j=1,2 .
\end{aligned}
$$

Of course, (3.18)-(3.25), (3.27)-(3.29), extend as usual to all $z \in \rho\left(H_{ \pm, 0, j}\right)$, respectively, all $z \in \rho\left(H_{j}\right), j=1,2$.

We note in passing that

$$
\begin{aligned}
& W\left(v_{j}(\bar{z}, \cdot)^{*}, u_{j}(z, \cdot)\right)(x) \text { is } x \text {-independent whenever } \\
& -\left[u_{j}^{\prime}(z, x)+(-1)^{j+1} \phi(x) u_{j}(z, x)\right]^{\prime}+(-1)^{j+1} \phi(x)\left[u_{j}^{\prime}(z, x)\right. \\
& \left.\quad+(-1)^{j+1} \phi(x) u_{j}(z, x)\right]=z u_{j}(z, x), \\
& -\left[v_{j}^{\prime}(\bar{z}, x)^{*}+(-1)^{j+1} v_{j}(\bar{z}, x)^{*} \phi(x)\right]^{\prime}+(-1)^{j+1}\left[v_{j}^{\prime}(\bar{z}, x)^{*}\right. \\
& \left.\quad+(-1)^{j+1} v_{j}(\bar{z}, x)^{*} \phi(x)\right] \phi(x)=z v_{j}(\bar{z}, x)^{*}, \quad j=1,2 .
\end{aligned}
$$

This circle of ideas will be further explored in Section 4

Remark 3.2. In the particular case where $\phi \in A C_{\text {loc }}(\mathbb{R})^{m \times m}$ and hence,

$$
V_{j}=\left[\phi^{2}+(-1)^{j} \phi^{\prime}\right] \in L_{\mathrm{loc}}^{1}(\mathbb{R})^{m \times m}, \quad j=1,2,
$$


the relation between the generalized Weyl-Titchmarsh matrices $\widehat{M}_{ \pm, 0, j}\left(\cdot, x_{0}\right)$, defined in (3.20), (3.21), and the standard (Dirichlet-type) Weyl-Titchmarsh matrices $M_{ \pm, 0, j}\left(\cdot, x_{0}\right)$ is especially simple and reads

$$
\widehat{M}_{ \pm, 0, j}\left(\cdot, x_{0}\right)=M_{ \pm, 0, j}\left(\cdot, x_{0}\right)+(-1)^{j+1} \phi\left(x_{0}\right), \quad j=1,2 .
$$

In particular, since $\phi\left(x_{0}\right)$ is $z$-independent and self-adjoint, the function theoretic (and hence spectral theoretic) content of $\widehat{M}_{ \pm, 0, j}\left(\cdot, x_{0}\right)$ and $M_{ \pm, 0, j}\left(\cdot, x_{0}\right)$ coincides in this special case as they possess identical matrix measures in their respective Nevanlinna-Herglotz representations. (These matrix measures being generated by the half-line $m \times m$ matrix-valued spectral functions of $H_{ \pm, 0, j}$.)

Remark 3.3. In the particular scalar case $m=1$, and under the stronger assumption $\phi \in L_{\text {loc }}^{2}(\mathbb{R})$, the operators $H_{j}, j=1,2$, and especially, the associated Miura transformation,

$$
\phi \mapsto \phi^{2}-\phi^{\prime}
$$

(i.e., the relation between $\phi$ and $V_{1}$ ), was studied in great detail in [77. However, the authors did not directly rely on $D$ and its supersymmetric structure, but instead based their investigations on an oscillation theoretic approach using Hartman's notion of (non)principal solutions. Subsequently, the authors of [35, 73], and 74] used a Zakharov-Shabat (ZS), or Ablowitz-Kaup-Newell-Segur (AKNS) Dirac-type expression $\widetilde{D}$ in connection with their investigation of Miura transformations and inverse scattering theory. Explicitly, $\widetilde{D}$ represents a self-adjoint $L^{2}(\mathbb{R})^{2}$-realization associated with the differential expression $\widetilde{\mathcal{D}}$ of the form

$$
\widetilde{\mathcal{D}}=i\left(\begin{array}{cc}
-(d / d x) & -\phi(x) \\
\phi(x) & (d / d x)
\end{array}\right) \text { for a.e. } x \in \mathbb{R} .
$$

To make the connection with the supersymmetric formalism presented in this paper, we introduce the unitary $2 \times 2$ matrices

$$
\Upsilon=\frac{1}{2}\left(\begin{array}{cc}
1-i & 1-i \\
1+i & -1-i
\end{array}\right), \quad \Upsilon^{*}=\frac{1}{2}\left(\begin{array}{cc}
1+i & 1-i \\
1+i & -1+i
\end{array}\right)=\Upsilon^{-1},
$$

and observe that

$$
\Upsilon \widetilde{\mathcal{D}} \Upsilon^{-1}=\mathcal{D}
$$

with $\mathcal{D}$ in (2.6) the differential expression underlying the supersymmetric Diractype operator $D$.

It is the use of supersymmetry of $D$ in connection with the (standard) assumption of local integrability of the coefficient $\phi$ that instantly leads to Weyl-Titchmarsh solutions $U_{ \pm}\left(\zeta, x, x_{0}, \alpha\right)$ and Weyl-Titchmarsh matrices $M_{ \pm}^{D}\left(\zeta, x_{0}, \alpha\right)$ of $D$ and hence effortlessly via (3.18) - (3.22) to those of the generalized Schrödinger-type operators $H_{ \pm, 0, j}, H_{j}, j=1,2$. In particular, it immediately leads to the Green's functions (3.27) - (3.29) of $H_{ \pm, 0, j}$ and of $H_{j}, j=1,2$, respectively, and permits the more general hypothesis $\phi \in L_{\text {loc }}^{1}(\mathbb{R})$ rather than $\phi \in L_{\text {loc }}^{2}(\mathbb{R})$. In addition, it permits an effortless discussion of the matrix-valued case (the latter cannot easily be obtained via oscillation theoretic methods, cf. the comments preceding [17, Hypothesis 3.6] in this context).

Remark 3.4. The supersymmetric formalism employed in this section relies on nonnegativity of $H_{1}=A^{*} A$ and $H_{2}=A A^{*}$ and so imposes a restriction on the distributional potential coefficients $V_{j}$ (formally, of the Miura-type $V_{j}(x)=$ $\left.\phi(x)^{2}+(-1)^{j} \phi^{\prime}(x)\right), j=1,2$. In practice, however, this restriction amounts to 
dealing with Schrödinger operators bounded from below as adding a sufficiently large positive constant to a given potential (if necessary) will render $H_{1}$ and $H_{2}$ nonnegative. (Subsequently, this additional constant can be removed.)

In this context we also notice that it is possible to generalize the operator $D$ in (2.5) to the form

$$
D_{m}=\left(\begin{array}{cc}
m & A^{*} \\
A & -m
\end{array}\right), \quad m \in \mathbb{R},
$$

to the effect that then

$$
D_{m}^{2}=\left(\begin{array}{cc}
A^{*} A+m^{2} I & 0 \\
0 & A A^{*}+m^{2} I
\end{array}\right)=\left(H_{1}+m^{2} I\right) \oplus\left(H_{2}+m^{2} I\right) .
$$

Finally, we note that these results on generalized Schrödinger operators with distributional potentials extend to general (three-coefficient) Sturm-Liouville operators, but we refrain from further details at this point.

\section{Basic Spectral Theory for $H_{j}, j=1,2$, and Some Applications}

In our final section we provide some spectral theoretic applications of the supersymmetric approach outlined in Section 3. In particular, upon deriving the basic aspects of Weyl-Titchmarsh theory for the generalized Schrödinger operators $H_{j}$, $j=1,2$, in $L^{2}(\mathbb{R})^{m}$ (cf. (3.5), (3.6)) and a discussion of the corresponding spectral representations, we derive a local Borg-Marchenko uniqueness theorem by utilizing the known analog for the Dirac operator $D$.

Since the Schrödinger operator $H_{2}$ as defined in (3.6) with coefficient $\phi(\cdot)$ is realized as a Schrödinger operator of the form $H_{1}$ as defined in (3.5) with coefficient $\phi(\cdot)$ replaced by $-\phi(\cdot)$, it suffices to exclusively study spectral theory for Schrödinger operators of the form $H_{1}$. In fact, the forms of many of the subsequent formulas relevant to the spectral theory of $H_{j}, j=1,2$, are independent of the choice $j \in\{1,2\}$, the exception being (4.35), (4.36) below.

To set the stage for the main results of this section, we begin with a discussion of solutions to the equation $\tau_{j} u=z u, z \in \mathbb{C} \backslash \mathbb{R}, j=1,2$, with $\tau_{j}$ as defined in (3.13), and their corresponding Wronskian relations.

To this end, suppose $z_{k} \in \mathbb{C} \backslash \mathbb{R}, k=1,2$, and that $u_{j}\left(z_{1}, \cdot\right)$ and $u_{j}\left(z_{2}, \cdot\right)$ satisfy

$$
u_{j}\left(z_{k}, \cdot\right), u_{j}^{[1, j]}\left(z_{k}, \cdot\right) \in A C_{\mathrm{loc}}(\mathbb{R})^{m \times m}, \quad \tau_{j} u_{j}\left(z_{k}, \cdot\right)=z_{k} u_{j}\left(z_{k}, \cdot\right), \quad j, k=1,2 .
$$

Then one observes that

$$
\begin{array}{r}
W\left(u_{j}\left(z_{1}, \cdot\right)^{*}, u_{j}\left(z_{2}, \cdot\right)\right)(x)=u_{j}\left(z_{1}, x\right)^{*} u_{j}^{[1, j]}\left(z_{2}, x\right)-\left(u_{j}^{[1, j]}\left(z_{1}, x\right)\right)^{*} u_{j}\left(z_{2}, x\right), \\
j=1,2,
\end{array}
$$

so that the Wronskian appearing in (4.2) is differentiable almost everywhere. Moreover, (4.1) implies

$$
\begin{array}{r}
\frac{d}{d x}\left(u_{j}^{[1, j]}\left(z_{k}, x\right)\right)=(-1)^{j+1} \phi(x) u_{j}^{[1, j]}\left(z_{k}, x\right)-z_{k} u_{j}\left(z_{k}, x\right) \\
\text { for a.e. } x \in \mathbb{R}, j, k=1,2 .
\end{array}
$$

As a result, one computes

$$
\begin{aligned}
& \frac{d}{d x} W\left(u_{j}\left(z_{1}, \cdot\right)^{*}, u_{j}\left(z_{2}, \cdot\right)\right)(x)=\frac{d}{d x}\left[u_{j}\left(z_{1}, x\right)^{*} u_{j}^{[1, j]}\left(z_{2}, x\right)-\left(u_{j}^{[1, j]}\left(z_{1}, x\right)\right)^{*} u_{j}\left(z_{2}, x\right)\right] \\
& \quad=\left(\overline{z_{1}}-z_{2}\right) u_{j}\left(z_{1}, x\right)^{*} u_{j}\left(z_{2}, x\right) \text { for a.e. } x \in \mathbb{R} .
\end{aligned}
$$


We summarize the above considerations as follows:

Lemma 4.1. Assume Hypothesis 2.1 and suppose that $z_{k} \in \mathbb{C} \backslash \mathbb{R}, k=1,2$. If $u_{j}\left(z_{k}, \cdot\right), j, k=1,2$, satisfy (4.1), then

$$
\begin{array}{r}
\frac{d}{d x} W\left(u_{j}\left(z_{1}, \cdot\right)^{*}, u_{j}\left(z_{2}, \cdot\right)\right)(x)=\left(\overline{z_{1}}-z_{2}\right) u_{j}\left(z_{1}, x\right)^{*} u_{j}\left(z_{2}, x\right) \\
\text { for a.e. } x \in \mathbb{R}, j=1,2 .
\end{array}
$$

As an immediate consequence of Lemma 4.1, one has the following result.

Corollary 4.2. Assume Hypothesis 2.1 . Then for $z \in \mathbb{C} \backslash \mathbb{R}$, the following identity holds.

$$
\begin{array}{r}
\operatorname{Im}(z) \int_{x_{0}}^{x} d x^{\prime} \psi_{ \pm, j}\left(z, x^{\prime}, x_{0}\right)^{*} \psi_{ \pm, j}\left(z, x^{\prime}, x_{0}\right) \\
=\operatorname{Im}\left(\widehat{M}_{ \pm, 0, j}\left(z, x_{0}\right)\right)-(2 i)^{-1} W\left(\psi_{ \pm, j}\left(z, \cdot, x_{0}\right)^{*}, \begin{array}{r}
\left.\psi_{ \pm, j}\left(z, \cdot, x_{0}\right)\right)(x) \\
x \in \mathbb{R}, j=1,2 .
\end{array}\right.
\end{array}
$$

Proof. Let $z \in \mathbb{C} \backslash \mathbb{R}$ be fixed and choose $u_{j}\left(z_{1}, \cdot\right)=u_{j}\left(z_{2}, \cdot\right)=\psi_{ \pm, j}\left(z, \cdot, x_{0}\right)$, $j=1,2$, in (4.5), one obtains

$$
\begin{aligned}
& \frac{d}{d x^{\prime}} W\left(\psi_{ \pm, j}\left(z, \cdot, x_{0}\right)^{*}, \psi_{ \pm, j}\left(z, \cdot, x_{0}\right)\right)\left(x^{\prime}\right) \\
& \quad=-2 i \operatorname{Im}(z) \psi_{ \pm, j}\left(z, x^{\prime}, x_{0}\right)^{*} \psi_{ \pm, j}\left(z, x^{\prime}, x_{0}\right) \text { for a.e. } x^{\prime} \in \mathbb{R}, j=1,2 .
\end{aligned}
$$

Integration of both sides of (4.7) from $x_{0}$ to $x$, using the normalizations in (3.23) and (3.24), yields (4.6).

Lemma 4.3. Assume Hypothesis 2.1. Then for any $z \in \mathbb{C} \backslash \mathbb{R}$,

$$
\lim _{x \rightarrow \pm \infty} W\left(\psi_{ \pm, j}\left(z, \cdot, x_{0}\right)^{*}, \psi_{ \pm, j}\left(z, \cdot, x_{0}\right)\right)(x)=0, \quad j=1,2 .
$$

Proof. We provide a proof of (4.8) for the case $j=1$; an analogous argument is used to settle the case $j=2$. In order to prove (4.8) for $j=1$, fix $z \in \mathbb{C} \backslash \mathbb{R}$ and observe that by (3.11) and (3.18),

$$
\begin{aligned}
W_{1}\left(\psi_{ \pm, 1}\left(z, \cdot, x_{0}\right)^{*}, \psi_{ \pm, 1}\left(z, \cdot, x_{0}\right)\right)(x) \\
=\psi_{ \pm, 1}\left(z, x, x_{0}\right)^{*} \psi_{ \pm, 1}^{[1,1]}\left(z, x, x_{0}\right)-\psi_{ \pm, 1}^{[1,1]}\left(z, x, x_{0}\right)^{*} \psi_{ \pm, 1}\left(z, x, x_{0}\right) \\
=\zeta u_{ \pm, 1}\left(\zeta, x, x_{0}, \alpha_{0}\right)^{*} u_{ \pm, 2}\left(\zeta, x, x_{0}, \alpha_{0}\right)-\zeta u_{ \pm, 2}\left(\zeta, x, x_{0}, \alpha_{0}\right)^{*} u_{ \pm, 1}\left(\zeta, x, x_{0}, \alpha_{0}\right) \\
=(\zeta-\bar{\zeta}) u_{ \pm, 2}\left(\zeta, x, x_{0}, \alpha_{0}\right)^{*} u_{ \pm, 1}\left(\zeta, x, x_{0}, \alpha_{0}\right) \\
\quad-\bar{\zeta} U_{ \pm}\left(\zeta, x, x_{0}, \alpha_{0}\right)^{*} J U_{ \pm}\left(\zeta, x, x_{0}, \alpha_{0}\right) \text { for a.e. } x \in \mathbb{R}, z=\zeta^{2} .
\end{aligned}
$$

Since $\mathcal{D}$ is in the limit point case one has the following limit relation (cf., e.g., 62 , Corollary 2.3])

$$
\lim _{x \rightarrow \pm \infty} U_{ \pm}\left(\zeta, x, x_{0}, \alpha_{0}\right)^{*} J U_{ \pm}\left(\zeta, x, x_{0}, \alpha_{0}\right)=0 .
$$

Thus, in order to prove (4.8), it suffices to show

$$
\lim _{x \rightarrow \pm \infty} u_{ \pm, 2}\left(\zeta, x, x_{0}, \alpha_{0}\right)^{*} u_{ \pm, 1}\left(\zeta, x, x_{0}, \alpha_{0}\right)=0 .
$$

To this end, one observes that the function under the limit in (4.11) is differentiable and that, in fact,

$$
\left[u_{ \pm, 2}\left(\zeta, \cdot, x_{0}, \alpha_{0}\right)^{*} u_{ \pm, 1}\left(\zeta, \cdot, x_{0}, \alpha_{0}\right)\right]^{\prime}(x)
$$




$$
\begin{array}{r}
=\zeta u_{ \pm, 2}\left(\zeta, x, x_{0}, \alpha_{0}\right)^{*} u_{ \pm, 2}\left(\zeta, x, x_{0}, \alpha_{0}\right)-\bar{\zeta} u_{ \pm, 1}\left(\zeta, x, x_{0}, \alpha_{0}\right)^{*} u_{ \pm, 1}\left(\zeta, x, x_{0}, \alpha_{0}\right) \\
\text { for a.e. } x \in \mathbb{R} .
\end{array}
$$

We recall that $u_{ \pm, 2}\left(\zeta, \cdot, x_{0}, \alpha_{0}\right), u_{ \pm, 1}\left(\zeta, \cdot, x_{0}, \alpha_{0}\right) \in L^{2}((0, \pm \infty))^{m \times m}$. As a result, one infers that

$$
u_{ \pm, 2}\left(\zeta, \cdot, x_{0}, \alpha_{0}\right)^{*} u_{ \pm, 1}\left(\zeta, \cdot x_{0}, \alpha_{0}\right) \in L^{1}((0, \pm \infty))^{m \times m}
$$

the same containment is true for the derivative by (4.12). Moreover,

$$
\begin{aligned}
& u_{ \pm, 2}\left(\zeta, x, x_{0}, \alpha_{0}\right)^{*} u_{ \pm, 1}\left(\zeta, x, x_{0}, \alpha_{0}\right)=u_{ \pm, 2}\left(\zeta, 0, x_{0}, \alpha_{0}\right)^{*} u_{ \pm, 1}\left(\zeta, 0, x_{0}, \alpha_{0}\right) \\
& +\int_{0}^{x} d x^{\prime}\left[u_{ \pm, 2}\left(\zeta, x^{\prime}, x_{0}, \alpha_{0}\right)^{*} u_{ \pm, 1}\left(\zeta, x^{\prime}, x_{0}, \alpha_{0}\right)\right]^{\prime}\left(x^{\prime}\right), \quad x \in \mathbb{R},
\end{aligned}
$$

coupled with the fact that the function appearing under the integral in (4.14) belongs to $L^{1}((0, \pm \infty))$, affirms the existence of the limits appearing in (4.11). In light of (4.13), both limits must equal zero.

Taking limits $x \rightarrow \pm \infty$ throughout (4.6) and using (4.8), one obtains the fundamental identities:

$$
\begin{array}{r}
\operatorname{Im}\left(\widehat{M}_{ \pm, 0, j}\left(z, x_{0}\right)\right)=\operatorname{Im}(z) \int_{x_{0}}^{ \pm \infty} d x^{\prime} \psi_{ \pm, j}\left(z, x^{\prime}, x_{0}\right)^{*} \psi_{ \pm, j}\left(z, x^{\prime}, x_{0}\right) \\
z \in \mathbb{C} \backslash \mathbb{R}, j=1,2 .
\end{array}
$$

The identities in (4.15) show that $\pm \widehat{M}_{ \pm, 0, j}\left(z, x_{0}\right), j=1,2$, are Nevanlinna-Herglotz functions. We summarize this together with some other relevant properties of the generalized Dirichlet-type $m \times m$ matrix-valued Weyl-Titchmarsh functions $\widehat{M}_{ \pm, 0, j}\left(z, x_{0}\right), j=1,2$, (associated to $H_{j}$ ) in the following result.

Lemma 4.4. Assume Hypothesis 2.1 and let $\widehat{M}_{ \pm, 0, j}\left(z, x_{0}\right), j=1,2, z \in \mathbb{C} \backslash \mathbb{R}$, denote the generalized Dirichlet-type $m \times m$ matrix-valued Weyl-Titchmarsh functions associated to $H_{j}$ as defined by (3.20) and (3.21). Then $\pm \widehat{M}_{ \pm, 0, j}\left(\cdot, x_{0}\right), j=1,2$, is an $m \times m$ matrix-valued Nevanlinna-Herglotz function of maximal rank $m$. In particular,

$$
\begin{aligned}
& \operatorname{Im}\left( \pm \widehat{M}_{ \pm, 0, j}\left(z, x_{0}\right)\right) \geqslant 0, \quad z \in \mathbb{C}_{+}, \\
& \widehat{M}_{ \pm, 0, j}\left(\bar{z}, x_{0}\right)=\widehat{M}_{ \pm, 0, j}\left(z, x_{0}\right)^{*}, \quad z \in \mathbb{C} \backslash \mathbb{R}, \\
& \operatorname{rank}\left(\widehat{M}_{ \pm, 0, j}\left(z, x_{0}\right)\right)=m, \quad z \in \mathbb{C} \backslash \mathbb{R}, \\
& \lim _{\varepsilon \downarrow 0} \widehat{M}_{ \pm, 0, j}\left(\lambda+i \varepsilon, x_{0}\right) \quad \text { exists for a.e. } \lambda \in \mathbb{R}, j=1,2 .
\end{aligned}
$$

Proof. The inequalities in (4.16) follow immediately from (4.15). By (3.20) and (3.21), $\widehat{M}_{ \pm, 0, j}\left(\cdot, x_{0}\right), j=1,2$, are analytic on $\mathbb{C} \backslash \mathbb{R}$, thus they are NevanlinnaHerglotz by (4.16). Relation (4.17) (resp., (4.18) follows from (3.20) and (3.21) via (2.18) (resp., (2.19)). Finally, (4.19) follows from the fact that $\pm \widehat{M}_{ \pm, 0, j}\left(\cdot, x_{0}\right)$, $j=1,2$, are Nevanlinna-Herglotz functions (cf., e.g., 47]). Alternatively, (4.19) can be immediately inferred from (3.20) and (3.21) together with (2.20).

Remark 4.5. Above, we used Corollary 4.2 and Lemma 4.3 to prove $\pm \widehat{M}_{ \pm, 0, j}\left(\cdot, x_{0}\right)$, $j=1,2$, are Nevanlinna-Herglotz functions. Alternatively, one can use the following approach based on computing the imaginary parts for $\pm \widehat{M}_{ \pm, 0, j}\left(\cdot, x_{0}\right), j=1,2$, 
directly using the known representations for $\operatorname{Im}\left(M_{ \pm}^{D}\left(\zeta, c_{0}, \alpha_{0}\right)\right)$ (cf., (2.26)). We briefly sketch how this approach is carried out.

Note that the system

$$
\mathcal{D} \Psi=\zeta \Psi, \quad \zeta \in \mathbb{C} \backslash \mathbb{R}
$$

can be recast as

$$
J \Psi^{\prime}=\left[\zeta I_{2 m}+B\right] \Psi, \quad \zeta \in \mathbb{C} \backslash \mathbb{R},
$$

where $J$ is the $2 m \times 2 m$ matrix defined in (2.7), and

$$
B=B(x)=\left(\begin{array}{cc}
0 & \phi(x) \\
\phi(x) & 0
\end{array}\right) \text { for a.e. } x \in \mathbb{R} .
$$

One can then verify by direct computation that if $\Psi_{j}=\Psi_{j}\left(\zeta_{j}, \cdot\right), j=1,2$, denote solutions of (4.21) with $\zeta_{j} \in \mathbb{C} \backslash \mathbb{R}, j=1,2$, then

$$
\left(\Psi_{1}^{*} \mathfrak{S}_{1} \Psi_{2}\right)^{\prime}=-\left(\zeta_{2}+\overline{\zeta_{1}}\right) \Psi_{1}^{*} \mathfrak{S}_{3} \Psi_{2}
$$

where

$$
\mathfrak{S}_{1}=\left(\begin{array}{cc}
0 & I_{m} \\
I_{m} & 0
\end{array}\right), \quad \mathfrak{S}_{3}=\left(\begin{array}{cc}
I_{m} & 0 \\
0 & -I_{m}
\end{array}\right)
$$

Let $z \in \mathbb{C} \backslash \mathbb{R}$ and fix $\zeta \in \mathbb{C}$ with $\zeta^{2}=z$ and $\operatorname{Im}(\zeta)>0$. Upon decomposing $\zeta$ and $\pm \widehat{M}_{ \pm, 0,1}\left(\cdot, x_{0}\right)$ into its real and imaginary parts and using (3.20), one computes

$$
\operatorname{Im}\left( \pm \widehat{M}_{ \pm, 0,1}\left(z, x_{0}\right)\right)=\operatorname{Im}(\zeta) \operatorname{Re}\left(M_{ \pm}^{D}\left(\zeta, x_{0}, \alpha_{0}\right)\right)+\operatorname{Re}(\zeta) \operatorname{Im}\left(M_{ \pm}^{D}\left(\zeta, x_{0}, \alpha_{0}\right)\right)
$$

Choosing $\Psi_{1}=\Psi_{2}=U_{ \pm}\left(\zeta, \cdot, x_{0}, \alpha_{0}\right)$ and $\zeta_{1}=\zeta_{2}=\zeta$ in (4.23) and integrating over $\left[x_{0}, \infty\right)$ yields

$$
\begin{gathered}
\operatorname{Re}\left(M_{ \pm}^{D}\left(\zeta, x_{0}, \alpha_{0}\right)\right)=\frac{1}{2} \lim _{x^{\prime} \rightarrow \pm \infty} U_{ \pm}\left(\zeta, x^{\prime}, x_{0}, \alpha_{0}^{*}\right) \mathfrak{S}_{1} U_{ \pm}\left(\zeta, x^{\prime}, x_{0}, \alpha_{0}\right) \\
+\operatorname{Re}(\zeta) \int_{x_{0}}^{ \pm \infty} d x\left[u_{ \pm, 1}\left(\zeta, x, x_{0}, \alpha_{0}\right)^{*} u_{ \pm, 1}\left(\zeta, x, x_{0}, \alpha_{0}\right)\right. \\
\left.\quad-u_{ \pm, 2}\left(\zeta, x, x_{0}, \alpha_{0}\right)^{*} u_{ \pm, 2}\left(\zeta, x, x_{0}, \alpha_{0}\right)\right] \\
=\operatorname{Re}(\zeta) \int_{x_{0}}^{ \pm \infty} d x\left[u_{ \pm, 1}\left(\zeta, x, x_{0}, \alpha_{0}\right)^{*} u_{ \pm, 1}\left(\zeta, x, x_{0}, \alpha_{0}\right)\right. \\
\left.\quad-u_{ \pm, 2}\left(\zeta, x, x_{0}, \alpha_{0}\right)^{*} u_{ \pm, 2}\left(\zeta, x, x_{0}, \alpha_{0}\right)\right]
\end{gathered}
$$

applying (4.11). The fact that the supersymmetric nature of $D$ permits the representation (4.27) for the real part of $M_{ \pm}^{D}\left(\zeta, x_{0}, \alpha_{0}\right)$ appears to have gone unnoticed in the literature. By (2.26), the representation in (4.25) can be recast as

$$
\begin{aligned}
\operatorname{Im}\left(\widehat{M}_{ \pm, 0,1}\left(z, x_{0}\right)\right) & =2 \operatorname{Im}(\zeta) \operatorname{Re}(\zeta) \int_{x_{0}}^{ \pm \infty} d x u_{ \pm, 1}\left(\zeta, x, x_{0}, \alpha_{0}\right)^{*} u_{ \pm, 1}\left(\zeta, x, x_{0}, \alpha_{0}\right) \\
& =\operatorname{Im}(z) \int_{x_{0}}^{ \pm \infty} d x \psi_{ \pm, 1}\left(z, x, x_{0}, \alpha_{0}\right)^{*} \psi_{ \pm, 1}\left(z, x, x_{0}, \alpha_{0}\right)
\end{aligned}
$$

(making use of (3.18) ), implying (4.15). The result for $\widehat{M}_{ \pm, 0,2}\left(z, x_{0}\right)$ follows similarly.

In order to establish spectral theory for $H_{j}$, we introduce the $2 m \times 2 m$ matrixvalued Weyl-Titchmarsh matrix, $\widehat{\mathbf{M}}_{j}\left(z, x_{0}\right) \in \mathbb{C}^{2 m \times 2 m}, z \in \mathbb{C} \backslash \mathbb{R}$, associated to $H_{j}$, $j=1,2$, as follows

$$
\widehat{\mathbf{M}}_{j}\left(z, x_{0}\right)=\left(\widehat{\mathbf{M}}_{j, k, k^{\prime}}\left(z, x_{0}\right)\right)_{k, k^{\prime}=0,1}, \quad z \in \mathbb{C} \backslash \mathbb{R}, j=1,2,
$$




$$
\begin{aligned}
& \widehat{\mathbf{M}}_{j, 0,0}\left(z, x_{0}\right)=W(z)^{-1}, \\
& \widehat{\mathbf{M}}_{j, 0,1}\left(z, x_{0}\right)=2^{-1} W(z)^{-1}\left[\widehat{M}_{-, 0, j}\left(z, x_{0}\right)+\widehat{M}_{+, 0, j}\left(z, x_{0}\right)\right], \\
& \widehat{\mathbf{M}}_{j, 1,0}\left(z, x_{0}\right)=2^{-1}\left[\widehat{M}_{-, 0, j}\left(z, x_{0}\right)+\widehat{M}_{+, 0, j}\left(z, x_{0}\right)\right] W(z)^{-1}, \\
& \widehat{\mathbf{M}}_{j, 1,1}\left(z, x_{0}\right)=\widehat{M}_{ \pm, 0, j}\left(z, x_{0}\right) W(z)^{-1} \widehat{M}_{\mp, 0, j}\left(z, x_{0}\right),
\end{aligned}
$$

where we have used the abbreviation (cf. (3.31))

$$
\begin{aligned}
W(z) & =W\left(\psi_{+, j}\left(\bar{z}, \cdot, x_{0}\right)^{*}, \psi_{-, j}\left(z, \cdot, x_{0}\right)\right) \\
& =\widehat{M}_{-, 0, j}\left(z, x_{0}\right)-\widehat{M}_{+, 0, j}\left(z, x_{0}\right), \quad z \in \mathbb{C} \backslash \mathbb{R}, j=1,2 .
\end{aligned}
$$

With (3.25) and the definitions in (4.29)-(4.33), one readily verifies that

$$
\begin{aligned}
\widehat{\mathbf{M}}_{1}\left(z, x_{0}\right)= & \left(\begin{array}{cc}
\zeta^{-1} I_{m} & 0 \\
0 & I_{m}
\end{array}\right) \mathbf{M}^{D}\left(\zeta, x_{0}, \alpha_{0}\right)\left(\begin{array}{cc}
I_{m} & 0 \\
0 & \zeta I_{m}
\end{array}\right), \\
\widehat{\mathbf{M}}_{2}\left(z, x_{0}\right)= & \left(\begin{array}{cc}
-\zeta^{-1} M_{+}^{D}\left(\zeta, x_{0}, \alpha_{0}\right) & M_{-}^{D}\left(\zeta, x_{0}, \alpha_{0}\right)^{-1}
\end{array}\right) \mathbf{M}^{D}\left(\zeta, x_{0}, \alpha_{0}\right) \\
0 & \times \\
& \times\left(\begin{array}{cc}
-M_{-}^{D}\left(\zeta, x_{0}, \alpha_{0}\right) & \\
0 & \zeta M_{+}^{D}\left(\zeta, x_{0}, \alpha_{0}\right)^{-1}
\end{array}\right), \quad \zeta^{2}=z, \zeta \in \mathbb{C} \backslash \mathbb{R} .
\end{aligned}
$$

In addition, one notes that $\widehat{\mathbf{M}}_{j}\left(z, x_{0}\right)$ is a $\mathbb{C}^{2 m \times 2 m}$-valued Nevanlinna-Herglotz matrix with representation

$$
\begin{aligned}
& \widehat{\mathbf{M}}_{j}\left(z, x_{0}\right)=\mathbf{C}_{j}\left(x_{0}\right)+\int_{\mathbb{R}} d \widehat{\boldsymbol{\Omega}}_{j}\left(\lambda, x_{0}\right)\left[\frac{1}{\lambda-z}-\frac{\lambda}{1+\lambda^{2}}\right], \quad z \in \mathbb{C} \backslash \mathbb{R}, \\
& \mathbf{F}_{j}\left(x_{0}\right)=\mathbf{F}_{j}\left(x_{0}\right)^{*}, \quad \int_{\mathbb{R}}\left\|d \widehat{\boldsymbol{\Omega}}_{j}\left(\lambda, x_{0}\right)\right\|_{\mathbb{C}^{2 m}}\left(1+\lambda^{2}\right)^{-1}<\infty, \quad j=1,2 .
\end{aligned}
$$

The Stieltjes inversion formula for the nonnegative $2 m \times 2 m$ matrix-valued measure $d \widehat{\boldsymbol{\Omega}}_{j}\left(\cdot, x_{0}\right)$ then reads

$$
\begin{array}{r}
\widehat{\boldsymbol{\Omega}}_{j}\left(\left(\lambda_{1}, \lambda_{2}\right], x_{0}\right)=\frac{1}{\pi} \lim _{\delta \downarrow 0} \lim _{\varepsilon \downarrow 0} \int_{\lambda_{1}+\delta}^{\lambda_{2}+\delta} d \lambda \operatorname{Im}\left(\widehat{\mathbf{M}}_{j}\left(\lambda+i \varepsilon, x_{0}\right)\right), \\
\lambda_{1}, \lambda_{2} \in \mathbb{R}, \lambda_{1}<\lambda_{2}, j=1,2 .
\end{array}
$$

In particular, $d \widehat{\boldsymbol{\Omega}}_{j}\left(\cdot, x_{0}\right), j=1,2$, is a $2 \times 2$ block matrix-valued measure with $\mathbb{C}^{m \times m}$-valued entries $d \widehat{\boldsymbol{\Omega}}_{j, \ell, \ell^{\prime}}\left(\cdot, x_{0}\right), \ell, \ell^{\prime}=0,1$. Since the diagonal entries of $\widehat{\mathbf{M}}_{j}\left(\cdot, x_{0}\right)$ are Nevanlinna-Herglotz functions, the diagonal entries of the measure $d \widehat{\boldsymbol{\Omega}}_{j}\left(\cdot, x_{0}\right)$ are nonnegative $\mathbb{C}^{m \times m}$-valued measures. The off-diagonal entries of the measure $d \widehat{\boldsymbol{\Omega}}_{j}\left(\cdot, x_{0}\right)$ naturally admit decompositions into a linear combination of four nonnegative matrix-valued measures.

Next, we relate the family of spectral projections $\left\{E_{H_{j}}(\lambda)\right\}_{\lambda \in \mathbb{R}}$ of the self-adjoint operator $H_{j}$ and the $2 m \times 2 m$ matrix-valued increasing spectral function $\widehat{\Omega}_{j}\left(\lambda, x_{0}\right)$, $\lambda \in \mathbb{R}$, which generates the matrix-valued measure in the Nevanlinna-Herglotz representation (4.37) of $\widehat{\mathbf{M}}_{j}\left(\cdot, x_{0}\right), j=1,2$.

We note that for $F \in C(\mathbb{R})$,

$$
\left(f, F\left(H_{j}\right) g\right)_{L^{2}(\mathbb{R})^{m}}=\int_{\mathbb{R}} d\left(f, E_{H_{j}}(\lambda) g\right)_{L^{2}(\mathbb{R})^{m}} F(\lambda),
$$




$$
\begin{array}{r}
f, g \in \operatorname{dom}\left(F\left(H_{j}\right)\right)=\left\{\left.h \in L^{2}(\mathbb{R})^{m}\left|\int_{\mathbb{R}} d\left\|E_{H_{j}}(\lambda) h\right\|_{L^{2}(\mathbb{R})^{m}}^{2}\right| F(\lambda)\right|^{2}<\infty\right\}, \\
j=1,2 .
\end{array}
$$

Theorem 4.6. Assume Hypothesis 2.1 and let $f, g \in C_{0}^{\infty}(\mathbb{R})^{m}, F \in C(\mathbb{R}), x_{0} \in \mathbb{R}$, and $\lambda_{1}, \lambda_{2} \in \mathbb{R}, \lambda_{1}<\lambda_{2}$. Then,

$$
\begin{aligned}
& \left(f, F\left(H_{j}\right) E_{H_{j}}\left(\left(\lambda_{1}, \lambda_{2}\right]\right) g\right)_{L^{2}(\mathbb{R})^{m}} \\
& \quad=\left(\widehat{f}_{j}\left(\cdot, x_{0}\right), M_{F} M_{\chi_{\left(\lambda_{1}, \lambda_{2}\right]}} \widehat{g}_{j}\left(\cdot, x_{0}\right)\right)_{L^{2}\left(\mathbb{R} ; d \widehat{\Omega}_{j}\left(\cdot, x_{0}\right)\right)}, \quad j=1,2,
\end{aligned}
$$

where we introduced the notation

$$
\begin{aligned}
& \widehat{h}_{j, 0}\left(\lambda, x_{0}\right)=\int_{\mathbb{R}} d x c_{j}\left(\lambda, x, x_{0}\right)^{*} h(x), \quad \widehat{h}_{j, 1}\left(\lambda, x_{0}\right)=\int_{\mathbb{R}} d x s_{j}\left(\lambda, x, x_{0}\right)^{*} h(x) \\
& \widehat{h}_{j}\left(\lambda, x_{0}\right)=\left(\widehat{h}_{j, 0}\left(\lambda, x_{0}\right), \widehat{h}_{j, 1}\left(\lambda, x_{0}\right)\right)^{\top}, \quad \lambda \in \mathbb{R}, h \in C_{0}^{\infty}(\mathbb{R})^{m}, \quad j=1,2,
\end{aligned}
$$

and $M_{G}$ denotes the maximally defined operator of multiplication by the function $G \in C(\mathbb{R})$ in the Hilbert space $L^{2}\left(\mathbb{R} ; d \widehat{\boldsymbol{\Omega}}_{j}\left(\cdot, x_{0}\right)\right)$,

$$
\begin{aligned}
& \left(M_{G} \widehat{h}\right)(\lambda)=G(\lambda) \widehat{h}(\lambda)=\left(G(\lambda) \widehat{h}_{0}(\lambda), G(\lambda) \widehat{h}_{1}(\lambda)\right)^{\top} \text { for } \widehat{\boldsymbol{\Omega}}_{j}\left(\cdot, x_{0}\right) \text {-a.e. } \lambda \in \mathbb{R}, \\
& \widehat{h} \in \operatorname{dom}\left(M_{G}\right)=\left\{\widehat{k} \in L^{2}\left(\mathbb{R} ; d \widehat{\boldsymbol{\Omega}}_{j}\left(\cdot, x_{0}\right)\right) \mid G \widehat{k} \in L^{2}\left(\mathbb{R} ; d \widehat{\boldsymbol{\Omega}}_{j}\left(\cdot, x_{0}\right)\right)\right\}, \quad j=1,2 .
\end{aligned}
$$

Proof. We fix $j \in\{1,2\}$. Using the weak version of Stone's formula, one obtains

$$
\begin{aligned}
\left(f, F\left(H_{j}\right) E_{H_{j}}\left(\left(\lambda_{1}, \lambda_{2}\right]\right) g\right)_{L^{2}(\mathbb{R})^{m}} & \\
=\lim _{\delta \downarrow 0} \lim _{\varepsilon \downarrow 0} \frac{1}{2 \pi i} \int_{\lambda_{1}+\delta}^{\lambda_{2}+\delta} d \lambda F(\lambda)[ & \left(f,\left(H_{j}-(\lambda+i \varepsilon) I_{L^{2}(\mathbb{R})^{m}}\right)^{-1} g\right)_{L^{2}(\mathbb{R})^{m}} \\
- & \left.\left(f,\left(H_{j}-(\lambda-i \varepsilon) I_{L^{2}(\mathbb{R})^{m}}\right)^{-1} g\right)_{L^{2}(\mathbb{R})^{m}}\right] .
\end{aligned}
$$

Using that the resolvent of $H_{j}$ is an integral operator with kernel (3.29) in (4.43), and freely interchanging the $d x$ and $d x^{\prime}$ integrals with the limits and the $d \lambda$ integral (since all integration domains are finite and all integrands are continuous), and employing the expressions (3.22) for $\psi_{ \pm, j}\left(z, x, x_{0}\right)$, one obtains

$$
\begin{aligned}
&\left(f, F\left(H_{j}\right) E_{H_{j}}\left(\left(\lambda_{1}, \lambda_{2}\right]\right) g\right)_{L^{2}(\mathbb{R})^{m}}=\int_{\mathbb{R}} d x\left(f(x),\left\{\int_{-\infty}^{x} d x^{\prime}\right.\right. \\
& \times \lim _{\delta \downarrow 0} \lim _{\varepsilon \downarrow 0} \frac{1}{2 \pi i} \int_{\lambda_{1}+\delta}^{\lambda_{2}+\delta} d \lambda F(\lambda)\left[\left[c_{j}\left(\lambda, x, x_{0}\right)+s_{j}\left(\lambda, x, x_{0}\right) \widehat{M}_{+, 0, j}\left(\lambda+i \varepsilon, x_{0}\right)\right]\right. \\
& \times W(\lambda+i \varepsilon)^{-1}\left[c_{j}\left(\lambda, x^{\prime}, x_{0}\right)^{*}+\widehat{M}_{-, 0, j}\left(\lambda+i \varepsilon, x_{0}\right) s_{j}\left(\lambda, x^{\prime}, x_{0}\right)^{*}\right] g\left(x^{\prime}\right) \\
&-\quad\left[c_{j}\left(\lambda, x, x_{0}\right)+s_{j}\left(\lambda, x, x_{0}\right) \widehat{M}_{+, 0, j}\left(\lambda-i \varepsilon, x_{0}\right)\right] \\
&\left.\quad \times W(\lambda-i \varepsilon)^{-1}\left[c_{j}\left(\lambda, x^{\prime}, x_{0}\right)^{*}+\widehat{M}_{-, 0, j}\left(\lambda-i \varepsilon, x_{0}\right) s_{j}\left(\lambda, x^{\prime}, x_{0}\right)^{*}\right] g\left(x^{\prime}\right)\right] \\
&+\int_{x}^{\infty} d x^{\prime} \lim _{\delta \downarrow 0} \lim _{\varepsilon \downarrow 0} \frac{1}{2 \pi i} \int_{\lambda_{1}+\delta}^{\lambda_{2}+\delta} d \lambda F(\lambda) \\
& \times[ {\left[c_{j}\left(\lambda, x, x_{0}\right)+s_{j}\left(\lambda, x, x_{0}\right) \widehat{M}_{-, 0, j}\left(\lambda+i \varepsilon, x_{0}\right)\right] } \\
& \times W(\lambda+i \varepsilon)^{-1}\left[c_{j}\left(\lambda, x^{\prime}, x_{0}\right)^{*}+\widehat{M}_{+, 0, j}\left(\lambda+i \varepsilon, x_{0}\right) s_{j}\left(\lambda, x^{\prime}, x_{0}\right)^{*}\right] g\left(x^{\prime}\right) \\
&- {\left[c_{j}\left(\lambda, x, x_{0}\right)+s_{j}\left(\lambda, x, x_{0}\right) \widehat{M}_{-, 0, j}\left(\lambda-i \varepsilon, x_{0}\right)\right] }
\end{aligned}
$$




$$
\begin{aligned}
\times & W(\lambda-i \varepsilon)^{-1}\left[c_{j}\left(\lambda, x^{\prime}, x_{0}\right)^{*}\right. \\
& \left.\left.\left.\left.\quad+\widehat{M}_{+, 0, j}\left(\lambda-i \varepsilon, x_{0}\right) s_{j}\left(\lambda, x^{\prime}, x_{0}\right)^{*}\right] g\left(x^{\prime}\right)\right]\right\}\right)_{\mathbb{C}^{m}} .
\end{aligned}
$$

Here we employed (4.17), the fact that for fixed $x \in \mathbb{R}, c_{j}\left(z, x, x_{0}\right)$ and $s_{j}\left(z, x, x_{0}\right)$ are entire with respect to $z$, that $c_{j}\left(z, \cdot, x_{0}\right), s_{j}\left(z, \cdot, x_{0}\right) \in A C_{\mathrm{loc}}(\mathbb{R} ; \mathcal{H})$, and hence that

$$
\begin{aligned}
& c_{j}\left(\lambda \pm i \varepsilon, x, x_{0}\right) \underset{\varepsilon \downarrow 0}{=} c_{j}\left(\lambda, x, x_{0}\right) \pm\left. i \varepsilon(d / d z) c_{j}\left(z, x, x_{0}\right)\right|_{z=\lambda}+O\left(\varepsilon^{2}\right), \\
& s_{j}\left(\lambda \pm i \varepsilon, x, x_{0}\right) \underset{\varepsilon \downarrow 0}{=} s_{j}\left(\lambda, x, x_{0}\right) \pm\left. i \varepsilon(d / d z) s_{j}\left(z, x, x_{0}\right)\right|_{z=\lambda}+O\left(\varepsilon^{2}\right),
\end{aligned}
$$

with $O\left(\varepsilon^{2}\right)$ being uniform with respect to $(\lambda, x)$ as long as $\lambda$ and $x$ vary in compact subsets of $\mathbb{R}$. Moreover, we used that

$$
\begin{aligned}
& \varepsilon\left\|\widehat{\mathbf{M}}_{j}\left(\lambda+i \varepsilon, x_{0}\right)\right\|_{\mathbb{C}^{2 m \times 2 m}} \leqslant C\left(\lambda_{1}, \lambda_{2}, \varepsilon_{0}, x_{0}\right), \quad \lambda \in\left[\lambda_{1}, \lambda_{2}\right], 0<\varepsilon \leqslant \varepsilon_{0}, \\
& \varepsilon\left\|\operatorname{Re}\left(\widehat{\mathbf{M}}_{j}\left(\lambda+i \varepsilon, x_{0}\right)\right)\right\|_{\mathbb{C}^{2 m \times 2 m}} \underset{\varepsilon \downarrow 0}{=} o(1), \quad \lambda \in \mathbb{R},
\end{aligned}
$$

since $\widehat{\mathbf{M}}_{j}\left(\cdot, x_{0}\right)$, are $\mathbb{C}^{2 m \times 2 m}$-valued Nevanlinna-Herglotz functions. Moreover, we utilized (4.17), (4.45), (4.46), and the elementary facts

$$
\begin{aligned}
& \operatorname{Im}\left[\widehat{M}_{ \pm, 0, j}\left(\lambda+i \varepsilon, x_{0}\right) W(\lambda+i \varepsilon)^{-1}\right] \\
& \quad=\frac{1}{2} \operatorname{Im}\left[\left[\widehat{M}_{-, 0, j}\left(\lambda+i \varepsilon, x_{0}\right)+\widehat{M}_{+, 0, j}\left(\lambda+i \varepsilon, x_{0}\right)\right] W(\lambda+i \varepsilon)^{-1}\right], \\
& \operatorname{Im}\left[W(\lambda+i \varepsilon)^{-1} \widehat{M}_{ \pm, 0, j}\left(\lambda+i \varepsilon, x_{0}\right)\right] \\
& \quad=\frac{1}{2} \operatorname{Im}\left[W(\lambda+i \varepsilon)^{-1}\left[\widehat{M}_{-, 0, j}\left(\lambda+i \varepsilon, x_{0}\right)+\widehat{M}_{+, 0, j}\left(\lambda+i \varepsilon, x_{0}\right)\right]\right], \quad \lambda \in \mathbb{R}, \varepsilon>0 .
\end{aligned}
$$

Collecting appropriate terms in (4.44) then yields

$$
\begin{aligned}
(f, & \left.F\left(H_{j}\right) E_{H}\left(\left(\lambda_{1}, \lambda_{2}\right]\right) g\right)_{L^{2}(\mathbb{R})^{m}}=\int_{\mathbb{R}} d x\left(f(x), \int_{\mathbb{R}} d x^{\prime} \lim _{\delta \downarrow 0} \lim _{\varepsilon \downarrow 0} \frac{1}{\pi} \int_{\lambda_{1}+\delta}^{\lambda_{2}+\delta} d \lambda F(\lambda)\right. \\
\times & \left\{c_{j}\left(\lambda, x, x_{0}\right) \operatorname{Im}\left[W(\lambda+i \varepsilon)^{-1}\right] c_{j}\left(\lambda, x^{\prime}, x_{0}\right)^{*}\right. \\
+ & 2^{-1} c_{j}\left(\lambda, x, x_{0}\right) \\
& \quad \times \operatorname{Im}\left[W(\lambda+i \varepsilon)^{-1}\left[\widehat{M}_{-, 0, j}\left(\lambda+i \varepsilon, x_{0}\right)+\widehat{M}_{+, 0, j}\left(\lambda+i \varepsilon, x_{0}\right)\right]\right] s_{j}\left(\lambda, x^{\prime}, x_{0}\right)^{*} \\
+ & 2^{-1} s_{j}\left(\lambda, x, x_{0}\right) \\
& \times \operatorname{Im}\left[\left[\widehat{M}_{-, 0, j}\left(\lambda+i \varepsilon, x_{0}\right)+\widehat{M}_{+, 0, j}\left(\lambda+i \varepsilon, x_{0}\right)\right] W(\lambda+i \varepsilon)^{-1}\right] c_{j}\left(\lambda, x^{\prime}, x_{0}\right)^{*} \\
+ & s_{j}\left(\lambda, x, x_{0}\right) \\
\quad & \times \operatorname{Im}\left[\widehat{M}_{-, 0, j}\left(\lambda+i \varepsilon, x_{0}\right) W(\lambda+i \varepsilon)^{-1}\right. \\
& \left.\left.\left.\quad \times \widehat{M}_{+, 0, j}\left(\lambda+i \varepsilon, x_{0}\right)\right] s_{j}\left(\lambda, x^{\prime}, x_{0}\right)^{*}\right\} g\left(x^{\prime}\right)\right)_{\mathbb{C}^{m}} .
\end{aligned}
$$

Since by (4.38) (for $\left.\ell, \ell^{\prime}=0,1\right)$

$$
\begin{aligned}
& \int_{\left(\lambda_{1}, \lambda_{2}\right]} d \widehat{\boldsymbol{\Omega}}_{j, \ell, \ell^{\prime}}\left(\lambda, x_{0}\right)=\widehat{\boldsymbol{\Omega}}_{j, \ell, \ell^{\prime}}\left(\left(\lambda_{1}, \lambda_{2}\right], x_{0}\right) \\
& =\lim _{\delta \downarrow} \lim _{\varepsilon \downarrow 0} \frac{1}{\pi} \int_{\lambda_{1}+\delta}^{\lambda_{2}+\delta} d \lambda \operatorname{Im}\left(\widehat{\mathbf{M}}_{j, \ell, \ell^{\prime}}\left(\lambda+i \varepsilon, x_{0}\right)\right),
\end{aligned}
$$


one also has (again for $\ell, \ell^{\prime} \in\{0,1\}$ )

$$
\begin{gathered}
\int_{\mathbb{R}} d \widehat{\Omega}_{j, \ell, \ell^{\prime}}\left(\lambda, x_{0}\right) h(\lambda)=\lim _{\varepsilon \downarrow 0} \frac{1}{\pi} \int_{\mathbb{R}} d \lambda \operatorname{Im}\left(\widehat{\mathbf{M}}_{j, \ell, \ell^{\prime}}\left(\lambda+i \varepsilon, x_{0}\right)\right) h(\lambda), \quad h \in C_{0}(\mathbb{R})^{m}, \\
\int_{\left(\lambda_{1}, \lambda_{2}\right]} d \widehat{\boldsymbol{\Omega}}_{j, \ell, \ell^{\prime}}\left(\lambda, x_{0}\right) k(\lambda)=\lim _{\delta \downarrow 0} \lim _{\varepsilon \downarrow 0} \frac{1}{\pi} \int_{\lambda_{1}+\delta}^{\lambda_{2}+\delta} d \lambda \operatorname{Im}\left(\widehat{\mathbf{M}}_{j, \ell, \ell^{\prime}}\left(\lambda+i \varepsilon, x_{0}\right)\right) k(\lambda), \\
k \in C(\mathbb{R})^{m} .
\end{gathered}
$$

Then using (4.29)-(4.33), (4.41), and interchanging the $d x, d x^{\prime}$ and $d \widehat{\boldsymbol{\Omega}}_{j, \ell, \ell^{\prime}}\left(\cdot, x_{0}\right)$, $\ell, \ell^{\prime}=0,1$, integrals once more, one concludes from (4.48) that

$$
\begin{aligned}
& \left(f, F\left(H_{j}\right) E_{H_{j}}\left(\left(\lambda_{1}, \lambda_{2}\right]\right) g\right)_{L^{2}(\mathbb{R})^{m}} \\
& \quad=\int_{\left(\lambda_{1}, \lambda_{2}\right]} F(\lambda)\left(\widehat{f}_{j}\left(\lambda, x_{0}\right), d \widehat{\boldsymbol{\Omega}}_{j}\left(\lambda, x_{0}\right) \widehat{g}_{j}\left(\lambda, x_{0}\right)\right)_{\mathbb{C}^{2 m}},
\end{aligned}
$$

implying (4.40).

Next, we improve on Theorem 4.6 and remove the compact support restrictions on $f$ and $g$ in the usual way, closely following and appropriately adapting the argument of [50, (2.46)-(2.67)]. This leads to a variant of the spectral theorem for (functions of) $H_{j}, j=1,2$. We consider the map

$$
\begin{aligned}
& \widetilde{U}_{j}\left(x_{0}\right):\left\{\begin{array}{l}
C_{0}^{\infty}(\mathbb{R})^{m} \rightarrow L^{2}\left(\mathbb{R} ; d \widehat{\boldsymbol{\Omega}}_{j}\left(\cdot, x_{0}\right)\right) \\
h \mapsto \widehat{h}_{j}\left(\cdot, x_{0}\right)=\left(\widehat{h}_{j, 0}\left(\lambda, x_{0}\right), \widehat{h}_{j, 1}\left(\lambda, x_{0}\right)\right)^{\top},
\end{array}\right. \\
& \widehat{h}_{j, 0}\left(\lambda, x_{0}\right)=\int_{\mathbb{R}} d x c_{j}\left(\lambda, x, x_{0}\right)^{*} h(x), \quad \widehat{h}_{j, 1}\left(\lambda, x_{0}\right)=\int_{\mathbb{R}} d x s_{j}\left(\lambda, x, x_{0}\right)^{*} h(x) .
\end{aligned}
$$

Taking $f=g, F=1, \lambda_{1} \downarrow-\infty$, and $\lambda_{2} \uparrow \infty$ in (4.40) then shows that $\widetilde{U}_{j}\left(x_{0}\right)$, $j=1,2$, are densely defined isometries in $L^{2}(\mathbb{R})^{m}$, which extend by continuity to isometries on $L^{2}(\mathbb{R})^{m}$. The latter are denoted by $U_{j}\left(x_{0}\right)$ and are defined by

$$
\begin{aligned}
& U_{j}\left(x_{0}\right):\left\{\begin{array}{l}
L^{2}(\mathbb{R})^{m} \rightarrow L^{2}\left(\mathbb{R} ; d \widehat{\boldsymbol{\Omega}}_{j}\left(\cdot, x_{0}\right)\right) \\
h \mapsto \widehat{h}_{j}\left(\cdot, x_{0}\right)=\left(\widehat{h}_{j, 0}\left(\cdot, x_{0}\right), \widehat{h}_{j, 1}\left(\cdot, x_{0}\right)\right)^{\top},
\end{array}\right. \\
& \widehat{h}_{j}\left(\cdot, x_{0}\right)=\left(\begin{array}{l}
\widehat{h}_{j, 0}\left(\cdot, x_{0}\right) \\
\widehat{h}_{j, 1}\left(\cdot, x_{0}\right)
\end{array}\right)=\text { l.i.m. } \cdot a \downarrow-\infty, b \uparrow \infty \\
& \left(\begin{array}{l}
\int_{q}^{b} d x c_{j}\left(\cdot, x, x_{0}\right)^{*} h(x) \\
\int_{a}^{b} d x s_{j}\left(\cdot, x, x_{0}\right)^{*} h(x)
\end{array}\right),
\end{aligned}
$$

where l.i.m. refers to the $L^{2}\left(\mathbb{R} ; d \widehat{\boldsymbol{\Omega}}_{j}\left(\cdot, x_{0}\right)\right)$-limit.

Theorem 4.7. Assume Hypothesis 2.1 and let $F \in C(\mathbb{R})$ and $x_{0} \in \mathbb{R}$. Then,

$$
U_{j}\left(x_{0}\right) F\left(H_{j}\right) U_{j}\left(x_{0}\right)^{-1}=M_{F}, \quad j=1,2,
$$

in $L^{2}\left(\mathbb{R} ; d \widehat{\boldsymbol{\Omega}}_{j}\left(\cdot, x_{0}\right)\right)($ cf. (4.42) $)$. Moreover,

$$
\sigma\left(H_{j}\right)=\operatorname{supp}\left(d \widehat{\boldsymbol{\Omega}}_{j}\left(\cdot, x_{0}\right)\right), \quad j=1,2,
$$

and the multiplicity of the spectrum of $H_{j}, j=1,2$, is at most equal to $2 \mathrm{~m}$. 
Proof. Again, we fix $j \in\{1,2\}$. One observes that the calculation in (4.52) yields

$$
\begin{array}{r}
\left(E_{H_{j}}\left(\left(\lambda_{1}, \lambda_{2}\right]\right) g\right)(x)=\int_{\left(\lambda_{1}, \lambda_{2}\right]}\left(c_{j}\left(\lambda, x, x_{0}\right), s_{j}\left(\lambda, x, x_{0}\right)\right) d \widehat{\boldsymbol{\Omega}}_{j}\left(\lambda, x_{0}\right) \widehat{g}_{j}\left(\lambda, x_{0}\right), \\
g \in C_{0}^{\infty}(\mathbb{R})^{m},
\end{array}
$$

and, as a result, extends to all $g \in L^{2}(\mathbb{R})^{m}$ by continuity. Moreover, taking $\lambda_{1} \downarrow-\infty$ and $\lambda_{2} \uparrow \infty$ in (4.57) and using the spectral family properties s- $\lim _{\lambda \downarrow-\infty} E_{H_{j}}(\lambda)=$ 0 , s-lim $\lambda_{\lambda \uparrow \infty} E_{H_{j}}(\lambda)=I_{L^{2}(\mathbb{R})^{m}}$, where $E_{H_{j}}(\lambda)=E_{H_{j}}((-\infty, \lambda]), \lambda \in \mathbb{R}$, then yields

$$
\begin{array}{r}
g(\cdot)=\text { l.i.m. } \mu_{1 \downarrow} \downarrow-\infty, \mu_{2} \uparrow \infty \int_{\left(\mu_{1}, \mu_{2}\right]}\left(c_{j}\left(\lambda, \cdot, x_{0}\right), s_{j}\left(\lambda, \cdot, x_{0}\right)\right) d \widehat{\boldsymbol{\Omega}}_{j}\left(\lambda, x_{0}\right), \\
\widehat{g}_{j}\left(\lambda, x_{0}\right), \quad g \in L^{2}(\mathbb{R})^{m},
\end{array}
$$

where l.i.m. here refers to the limit in $L^{2}(\mathbb{R})^{m}$. Next, we show that the maps $U_{j}\left(x_{0}\right)$ in (4.54) are onto and hence that $U_{j}\left(x_{0}\right)$ are unitary maps with

$$
\begin{aligned}
& U_{j}\left(x_{0}\right)^{-1}:\left\{\begin{array}{l}
L^{2}\left(\mathbb{R} ; d \widehat{\mathbf{\Omega}}_{j}\left(\cdot, x_{0}\right)\right) \rightarrow L^{2}(\mathbb{R})^{m} \\
\widehat{h} \mapsto h_{j},
\end{array}\right. \\
& h_{j}(\cdot)=\text { l.i.m. } \mu_{1 \downarrow-\infty, \mu_{2} \uparrow \infty} \int_{\left(\mu_{1}, \mu_{2}\right]}\left(c_{j}\left(\lambda, \cdot, x_{0}\right), s_{j}\left(\lambda, \cdot, x_{0}\right)\right) d \widehat{\boldsymbol{\Omega}}_{j}\left(\lambda, x_{0}\right) \widehat{h}(\lambda) .
\end{aligned}
$$

Letting $W_{j}\left(x_{0}\right)$ temporarily denote the operators defined by (4.59), one infers that $W_{j}\left(x_{0}\right)$ are bounded. Indeed, for any $\widehat{f} \in C_{0}^{\infty}(\mathbb{R})^{2 m}, g \in C_{0}^{\infty}(\mathbb{R})^{m}$, one computes

$$
\begin{aligned}
(g & \left.W_{j}\left(x_{0}\right) \widehat{f}\right)_{L^{2}(\mathbb{R})^{m}} \\
& =\int_{\mathbb{R}} d x\left(g(x), \int_{\mathbb{R}}\left(c_{j}\left(\lambda, x, x_{0}\right), s_{j}\left(\lambda, x, x_{0}\right)\right) d \widehat{\boldsymbol{\Omega}}_{j}\left(\lambda, x_{0}\right) \widehat{f}(\lambda)\right)_{\mathbb{C}^{m}} \\
& =\int_{\mathbb{R}} \int_{\mathbb{R}} d x\left(\left(c_{j}\left(\lambda, x, x_{0}\right), s_{j}\left(\lambda, x, x_{0}\right)\right)^{*} g(x), d \widehat{\boldsymbol{\Omega}}_{j}\left(\lambda, x_{0}\right) \widehat{f}(\lambda)\right)_{\mathbb{C}^{2 m}} \\
& =\left(U_{j}\left(x_{0}\right) g, \widehat{f}\right)_{L^{2}\left(\mathbb{R} ; d \widehat{\boldsymbol{\Omega}}_{j}\left(\cdot, x_{0}\right)\right)} .
\end{aligned}
$$

Since $U_{j}\left(x_{0}\right)$ are isometries, (4.60) extends by continuity to all $g \in L^{2}(\mathbb{R})^{m}$. Thus,

$$
\begin{aligned}
\left\|W_{j}\left(x_{0}\right) \widehat{f}\right\|_{L^{2}(\mathbb{R})^{m}} & =\sup _{g \in L^{2}(\mathbb{R})^{m}, g \neq 0}\left|\frac{\left(g, W_{j}\left(x_{0}\right) \widehat{f}\right)_{L^{2}(\mathbb{R})^{m}}}{\|g\|_{L^{2}(\mathbb{R})^{m}}}\right| \\
& \leqslant \sup _{g \in L^{2}(\mathbb{R})^{m}, g \neq 0} \frac{\left\|U_{j}\left(x_{0}\right) g\right\|_{L^{2}\left(\mathbb{R} ; d \widehat{\boldsymbol{\Omega}}_{j}\left(\cdot, x_{0}\right)\right)}}{\|g\|_{L^{2}(\mathbb{R})^{m}}}\|\widehat{f}\|_{L^{2}\left(\mathbb{R} ; d \widehat{\boldsymbol{\Omega}}_{j}\left(\cdot, x_{0}\right)\right)} \\
& =\|\widehat{f}\|_{L^{2}\left(\mathbb{R} ; d \widehat{\boldsymbol{\Omega}}_{j}\left(\cdot, x_{0}\right)\right)}, \quad \widehat{f} \in C_{0}^{\infty}(\mathbb{R})^{m} .
\end{aligned}
$$

From the limiting relation in (4.59), one also infers that

$$
W_{j}\left(x_{0}\right) U_{j}\left(x_{0}\right)=I_{L^{2}(\mathbb{R})^{m}} \text {. }
$$

To verify that $U_{j}\left(x_{0}\right), j=1,2$, are onto, and hence unitary, it suffices to prove that $W_{j}\left(x_{0}\right)$ are injective. Suppose that $\widehat{f}=\left(f_{0}, f_{1}\right)^{\top} \in \operatorname{ker}\left(W_{j}\left(x_{0}\right)\right)$. Let $\widehat{f} \in$ $L^{2}\left(\mathbb{R} ; \widehat{\boldsymbol{\Omega}}_{j}\left(\cdot, x_{0}\right)\right), \lambda_{1}, \lambda_{2} \in \mathbb{R}, \lambda_{1}<\lambda_{2}$, and consider

$$
\left(H_{j}-z I_{L^{2}(\mathbb{R})^{m}}\right)\left(\int_{\left(\lambda_{1}, \lambda_{2}\right]}\left(c_{j}\left(\lambda, \cdot, x_{0}\right), s_{j}\left(\lambda, \cdot, x_{0}\right)\right)(\lambda-z)^{-1} d \widehat{\mathbf{\Omega}}_{j}\left(\lambda, x_{0}\right) \widehat{f}(\lambda)\right)
$$




$$
=\int_{\left(\lambda_{1}, \lambda_{2}\right]}\left(c_{j}\left(\lambda, \cdot, x_{0}\right), s_{j}\left(\lambda, \cdot, x_{0}\right)\right) d \widehat{\mathbf{\Omega}}_{j}\left(\lambda, x_{0}\right) \widehat{f}(\lambda), \quad z \in \mathbb{C}_{+} .
$$

Then,

$$
\begin{array}{r}
\int_{\left(\lambda_{1}, \lambda_{2}\right]}\left(c_{j}\left(\lambda, \cdot, x_{0}\right), s_{j}\left(\lambda, \cdot, x_{0}\right)\right)(\lambda-z)^{-1} d \widehat{\boldsymbol{\Omega}}_{j}\left(\lambda, x_{0}\right) \widehat{f}(\lambda) \\
=\left(H_{j}-z I_{L^{2}(\mathbb{R})^{m}}\right)^{-1}\left(\int_{\left(\lambda_{1}, \lambda_{2}\right]}\left(c_{j}\left(\lambda, \cdot, x_{0}\right), s_{j}\left(\lambda, \cdot, x_{0}\right)\right) d \widehat{\boldsymbol{\Omega}}_{j}\left(\lambda, x_{0}\right) \widehat{f}(\lambda)\right), \\
z \in \mathbb{C}_{+} .
\end{array}
$$

Taking s-lim $\lambda_{\lambda_{1} \downarrow-\infty, \lambda_{2} \uparrow \infty}$ in (4.64) implies

$$
W_{j}\left(x_{0}\right)\left((\cdot-z)^{-1} \widehat{f}\right)=\left(H_{j}-z I_{L^{2}\left((\mathbb{R})^{m}\right.}\right)^{-1} W_{j}\left(x_{0}\right) \widehat{f}, \quad z \in \mathbb{C}_{+} .
$$

Next, suppose that $\widehat{f}_{0}=\left(f_{0}, f_{1}\right)^{\top} \in \operatorname{ker}\left(W_{j}\left(x_{0}\right)\right)$, and take a sequence $\left\{\widehat{f}_{n}\right\}_{n \in \mathbb{N}} \subset$ $L^{2}\left(\mathbb{R} ; d \widehat{\boldsymbol{\Omega}}_{j}\left(\cdot, x_{0}\right)\right)$ such that $\operatorname{supp}\left(\widehat{f}_{n}\right)$ is compact for each $n \in \mathbb{N}$ and $\lim _{n \uparrow \infty} \| \widehat{f}_{0}-$ $\widehat{f}_{n} \|_{L^{2}\left(\mathbb{R} ; d \widehat{\boldsymbol{\Omega}}_{j}\left(\cdot, x_{0}\right)\right)}=0$. Then, since each $\widehat{f}_{n}$ is compactly supported,

$$
\begin{array}{r}
\left(W_{j}\left(x_{0}\right)\left((\cdot-z)^{-1} \widehat{f}_{n}\right)\right)(x)=\left(\left(H_{j}-z I_{L^{2}(\mathbb{R})^{m}}\right)^{-1} W_{j}\left(x_{0}\right) \widehat{f}_{n}\right)(x), \\
x \in \mathbb{R}, z \in \mathbb{C}_{+}, n \in \mathbb{N} .
\end{array}
$$

Consequently, for each $y \in \mathbb{R}$ and all $e \in \mathbb{C}^{m}$,

$$
\begin{array}{rl}
\int_{x_{0}}^{y} & d x \int_{\mathbb{R}}\left(e,\left(c_{j}\left(\lambda, x, x_{0}\right), s_{j}\left(\lambda, x, x_{0}\right)\right)(\lambda-z)^{-1} d \widehat{\boldsymbol{\Omega}}_{j}\left(\lambda, x_{0}\right) \widehat{f}_{n}(\lambda)\right)_{\mathbb{C}^{m}} \\
= & \int_{\mathbb{R}}\left(\int_{x_{0}}^{y} d x\left(c_{j}\left(\lambda, x, x_{0}\right), s_{j}\left(\lambda, x, x_{0}\right)\right)^{*} e, d \widehat{\boldsymbol{\Omega}}_{j}\left(\lambda, x_{0}\right)(\lambda-z)^{-1} \widehat{f}_{n}(\lambda)\right)_{\mathbb{C}^{m}} \\
= & \int_{x_{0}}^{y} d x\left(e,\left(\left(H_{j}-z I_{L^{2}(\mathbb{R})^{m}}\right)^{-1} W_{j}\left(x_{0}\right) \widehat{f}_{n}\right)(x)\right)_{\mathbb{C}^{m}} .
\end{array}
$$

One observes that

$$
\begin{aligned}
& \int_{x_{0}}^{\infty} d x\left(c_{j}\left(\lambda, x, x_{0}\right), s_{j}\left(\lambda, x, x_{0}\right)\right)^{*} \chi_{\left[x_{0}, y\right]}(x) e \\
& \quad=\left(U_{j}\left(x_{0}\right) \chi_{\left[x_{0}, y\right]} e\right)(\cdot) \in L^{2}\left(\mathbb{R} ; d \widehat{\boldsymbol{\Omega}}_{j}\left(\cdot, x_{0}\right)\right) .
\end{aligned}
$$

Thus, taking the limit $n \uparrow \infty$ in (4.66) yields

$$
\begin{aligned}
& \lim _{n \uparrow \infty} \int_{x_{0}}^{y} d x \int_{\mathbb{R}}\left(e,\left(c_{j}\left(\lambda, x, x_{0}\right), s_{j}\left(\lambda, x, x_{0}\right)\right) d \widehat{\boldsymbol{\Omega}}_{j}\left(\lambda, x_{0}\right)(\lambda-z)^{-1} \widehat{f}_{n}(\lambda)\right)_{\mathbb{C}^{m}} \\
& =\int_{\mathbb{R}}(\lambda-z)^{-1} \int_{x_{0}}^{y} d x\left(e,\left(c_{j}\left(\lambda, x, x_{0}\right), s_{j}\left(\lambda, x, x_{0}\right)\right) d \widehat{\boldsymbol{\Omega}}_{j}\left(\lambda, x_{0}\right) \widehat{f}_{0}(\lambda)\right)_{\mathbb{C}^{m}} \\
& =\lim _{n \uparrow \infty} \int_{x_{0}}^{y} d x\left(e,\left(\left(H_{j}-z I_{L^{2}\left((\mathbb{R})^{m}\right.}\right)^{-1} W_{j}\left(x_{0}\right) \widehat{f}_{n}\right)(x)\right)_{\mathbb{C}^{m}} \\
& =\int_{x_{0}}^{y} d x\left(e,\left(\left(H_{j}-z I_{L^{2}(\mathbb{R})^{m}}\right)^{-1} W_{j}\left(x_{0}\right) \widehat{f}_{0}\right)(x)\right)_{\mathbb{C}^{m}}=0, \\
& y \in \mathbb{R}, z \in \mathbb{C}_{+}, e \in \mathbb{C}^{m} .
\end{aligned}
$$


Applying the Stieltjes inversion formula to the (finite) complex-valued measure in the 3rd line of (4.69), given by,

$$
\int_{x_{0}}^{y} d x\left(e,\left(c_{j}\left(\lambda, x, x_{0}\right), s_{j}\left(\lambda, x, x_{0}\right)\right) d \widehat{\boldsymbol{\Omega}}_{j}\left(\lambda, x_{0}\right) \widehat{f}_{0}(\lambda)\right)_{\mathbb{C}^{m}},
$$

implies for all $\lambda_{1}, \lambda_{2} \in \mathbb{R}, \lambda_{1}<\lambda_{2}$, and $e \in \mathbb{C}^{m}$,

$$
\int_{\left(\lambda_{1}, \lambda_{2}\right]} \int_{x_{0}}^{y} d x\left(e,\left(c_{j}\left(\lambda, x, x_{0}\right), s_{j}\left(\lambda, x, x_{0}\right)\right) d \widehat{\boldsymbol{\Omega}}_{j}\left(\lambda, x_{0}\right) \widehat{f}_{0}(\lambda)\right)_{\mathbb{C}^{m}}=0, \quad y \in \mathbb{R} .
$$

Differentiating (4.71) with respect to $y$, noting that $c_{j}\left(\lambda, y, x_{0}\right)$ and $s_{j}\left(\lambda, y, x_{0}\right)$ are continuous in $(\lambda, y) \in \mathbb{R}^{2}$, and using the dominated convergence theorem, one obtains

$$
\int_{\left(\lambda_{1}, \lambda_{2}\right]}\left(e,\left(c_{j}\left(\lambda, y, x_{0}\right), s_{j}\left(\lambda, y, x_{0}\right)\right) d \widehat{\boldsymbol{\Omega}}_{j}\left(\lambda, x_{0}\right) \widehat{f}_{0}(\lambda)\right)_{\mathbb{C}^{m}}=0, \quad y \in \mathbb{R}, e \in \mathbb{C}^{m}
$$

In particular, taking $y=x_{0}$ in (4.72) and using (3.14) and (3.15), one obtains

$$
\begin{aligned}
& \int_{\left(\lambda_{1}, \lambda_{2}\right]}\left(e_{1},\left(c_{j}\left(\lambda, x_{0}, x_{0}\right), s_{j}\left(\lambda, x_{0}, x_{0}\right)\right) d \widehat{\boldsymbol{\Omega}}_{j}\left(\lambda, x_{0}\right) \widehat{f}_{0}(\lambda)\right)_{\mathbb{C}^{m}} \\
& \quad=\int_{\left(\lambda_{1}, \lambda_{2}\right]}\left(\left(e_{1}, 0_{m}\right)^{\top}, d \widehat{\boldsymbol{\Omega}}_{j}\left(\lambda, x_{0}\right) \widehat{f}_{0}(\lambda)\right)_{\mathbb{C}^{2 m}}=0, \quad e_{1} \in \mathbb{C}^{m},
\end{aligned}
$$

where $0_{m}$ in (4.73) denotes the zero vector in $\mathbb{C}^{m}$. Next, applying the quasiderivative $[1, j]$ with respect to $y \in \mathbb{R}$ to (4.72), yields

$$
\begin{array}{r}
\int_{\left(\lambda_{1}, \lambda_{2}\right]}\left(e,\left(c_{j}^{[1, j]}\left(\lambda, y, x_{0}\right), s_{j}^{[1, j]}\left(\lambda, y, x_{0}\right)\right) d \widehat{\boldsymbol{\Omega}}_{j}\left(\lambda, x_{0}\right) \widehat{f}_{0}(\lambda)\right)_{\mathbb{C}^{m}}=0, \\
y \in \mathbb{R}, e \in \mathbb{C}^{m},
\end{array}
$$

using the fact that

$$
\begin{array}{r}
\int_{\left(\lambda_{1}, \lambda_{2}\right]}\left(e,\left(\phi(y) c_{j}\left(\lambda, y, x_{0}\right), \phi(y) s_{j}\left(\lambda, y, x_{0}\right)\right) d \widehat{\boldsymbol{\Omega}}_{j}\left(\lambda, x_{0}\right) \widehat{f}_{0}(\lambda)\right)_{\mathbb{C}^{m}}=0, \\
y \in \mathbb{R}, e \in \mathbb{C}^{m} .
\end{array}
$$

Subsequently, taking $y=x_{0}$ in (4.74), once more using (3.14) and (3.15) yields

$$
\int_{\left(\lambda_{1}, \lambda_{2}\right]}\left(\left(0_{m}, e_{2}\right)^{\top}, d \widehat{\boldsymbol{\Omega}}_{j}\left(\lambda, x_{0}\right) \widehat{f}_{0}(\lambda)\right)_{\mathbb{C}^{2 m}}=0, \quad e_{2} \in \mathbb{C}^{m} .
$$

Taking $e=\left(e_{1}, e_{2}\right)^{\top}$ with $e_{1}, e_{2} \in \mathbb{C}^{m}$ and adding (4.73) and (4.76), one obtains

$$
\int_{\left(\lambda_{1}, \lambda_{2}\right]}\left(e, d \widehat{\boldsymbol{\Omega}}_{j}\left(\lambda, x_{0}\right) \widehat{f}_{0}(\lambda)\right)_{\mathbb{C}^{2 m}}=0, \quad e \in \mathbb{C}^{2 m} .
$$

Since $\lambda_{1}$ and $\lambda_{2}$ are arbitrary (apart from $\lambda_{1}<\lambda_{2}$ ), (4.77) implies

$$
\widehat{f}_{0}(\lambda)=0 \text { for } d \widehat{\boldsymbol{\Omega}}_{j}\left(\cdot, x_{0}\right) \text {-a.e. } \lambda \in \mathbb{R} .
$$

The proofs of Theorems 4.6 and 4.7 are adaptation of the proofs of Theorems 2.12 and 2.14 in $[50$. This strategy of proof immediately extends to all continuous or discrete second-order problems (such as Sturm-Liouville, Jacobi, and CMV operators) 
and first-order $2 \times 2$ systems (i.e., Dirac-type operators) with matrix-valued coefficients, see, for instance, [18, [19]. In fact, it also extends to the infinite-dimensional case of bounded operator-valued coefficients (for the case of Schrödinger operators with bounded operator-valued potentials, see [49]).

In our final result, we show that the known local Borg-Marchenko results for Dirac-type operators worked out in [16] immediately imply local Borg-Marchenko results for generalized Schrödinger operators of the form $H_{j}, j=1,2$. For simplicity, we focus on $H_{1}$ only.

Theorem 4.8. Suppose Hypothesis 2.1 holds with $\phi_{j}, j=1,2$, in place of $\phi$. Let $H_{1,1}$ (resp., $H_{1,2}$ ) denote the operator defined in (3.5) taking $\phi=\phi_{1}$ (resp., $\left.\phi=\phi_{2}\right)$ and denote by $\widehat{\mathbf{M}}_{1,1}\left(z, x_{0}\right)$ (resp., $\widehat{\mathbf{M}}_{1,2}\left(z, x_{0}\right)$ ) the corresponding $2 m \times 2 m$ block Weyl-Titchmarsh matrix as defined in (4.29)-(4.33). Then,

$$
\text { if for some } a>0, \phi_{1}(x)=\phi_{2}(x) \text { for a.e. } x \in\left(x_{0}-a, x_{0}+a\right) \text {, }
$$

one obtains

$$
\left\|\widehat{\mathbf{M}}_{1,1}\left(z, x_{0}\right)-\widehat{\mathbf{M}}_{1,2}\left(z, x_{0}\right)\right\|_{\mathbb{C}^{2 m \times 2 m}} \underset{\substack{|z| \rightarrow \infty \\ z \in \rho_{\theta}}}{=} O\left(z^{1 / 2} e^{-2 \operatorname{Im}\left(z^{1 / 2}\right) a}\right)
$$

along any ray $\rho_{\theta} \subset \mathbb{C}$ with $\arg (z)=\theta \in(0, \pi) \cup(\pi, 2 \pi)$, always choosing the branch of the square root with $\operatorname{Im}\left(z^{1 / 2}\right)>0$ for $z \in \mathbb{C} \backslash[0, \infty)$. On the other hand, suppose that for all $\varepsilon>0$,

$$
\left\|\widehat{\mathbf{M}}_{1,1}\left(z, x_{0}\right)-\widehat{\mathbf{M}}_{1,2}\left(z, x_{0}\right)\right\|_{\mathbb{C}^{2 m \times 2 m}} \underset{\substack{|z| \rightarrow \infty \\ z \in \rho_{\theta_{\ell}}}}{=} O\left(z^{1 / 2} e^{-2 \operatorname{Im}\left(z^{1 / 2}\right)(a-\varepsilon)}\right), \quad \ell=1,2,
$$

along a ray $\rho_{\theta_{1}} \subset \mathbb{C}$ with $\arg (z)=\theta_{1}$ and $0<\theta_{1}<\pi$ and along a ray $\rho_{\theta_{2}} \subset \mathbb{C}$ with $\arg (z)=\theta_{2}$ and $\pi<\theta_{2}<2 \pi$. If $m>1$, assume in addition that $\phi_{j} \in$ $L^{\infty}\left(\left[x_{0}-a, x_{0}+a\right]\right)^{m \times m}, j=1,2$. Then

$$
\phi_{1}(x)=\phi_{2}(x) \text { for a.e. } x \in\left[x_{0}-a, x_{0}+a\right] .
$$

Proof. We begin by fixing some notation. Let $\mathbf{M}_{j}^{D}\left(\zeta, x_{0}, \alpha_{0}\right), j=1,2$, denote the $2 m \times 2 m$ Weyl-Titchmarsh matrix defined by (2.30) corresponding to the Diractype operator $D_{j}, j=1,2$ defined by (2.5), that is, the operator (formally) defined as

$$
D_{j}=J \frac{d}{d x}-B_{j}(x), \quad B_{j}(x)=\left(\begin{array}{cc}
0 & -\phi_{j}(x) \\
-\phi_{j}(x) & 0
\end{array}\right), j=1,2 .
$$

As a result of (4.35), one estimates

$$
\begin{array}{r}
\left\|\widehat{\mathbf{M}}_{1,1}\left(z, x_{0}\right)-\widehat{\mathbf{M}}_{1,2}\left(z, x_{0}\right)\right\|_{\mathbb{C}^{2 m \times 2 m}} \\
\leqslant C|\zeta|\left\|\mathbf{M}_{1}^{D}\left(\zeta, x_{0}, \alpha_{0}\right)-\mathbf{M}_{2}^{D}\left(\zeta, x_{0}, \alpha_{0}\right)\right\|_{\mathbb{C}^{2 m \times 2 m}}, \\
\zeta^{2}=z \in \mathbb{C} \backslash \mathbb{R},|z| \geqslant 1
\end{array}
$$

for a $z$-independent constant $C>0$.

Evidently, taking $|z| \rightarrow \infty$ along the fixed ray $\rho_{\theta} \subset \mathbb{C}$ with $\arg (z)=\theta$ and $\theta \in(0, \pi) \cup(\pi, 2 \pi)$ implies

$|\zeta| \rightarrow \infty$, along the fixed ray $\rho_{\theta / 2} \subset \mathbb{C}_{+}$with $0<\arg (\zeta)=\theta / 2<\pi$,

if $\zeta^{2}=z$. The assumption in (4.79) implies

$$
B_{1}(x)=B_{2}(x) \text { for a.e. } x \in\left(x_{0}-a, x_{0}+a\right),
$$


with $B_{j}(\cdot), j=1,2$, as in (4.83). Consequently, [16, Theorem 5.5] implies

$$
\left\|\mathbf{M}_{1}^{D}\left(\zeta, x_{0}, \alpha_{0}\right)-\mathbf{M}_{2}^{D}\left(\zeta, x_{0}, \alpha_{0}\right)\right\|_{\mathbb{C}^{2 m \times 2 m}} \underset{\substack{|\zeta| \rightarrow \infty \\ \zeta \in \rho_{\theta / 2}}}{=} O\left(e^{-2 \operatorname{Im}(\zeta) a}\right) .
$$

As $\operatorname{Re}(\zeta)=2 \theta^{-1} \operatorname{Im}(\zeta)$ for $\zeta \in \rho_{\theta / 2}$, (4.87) implies

$$
|\zeta|\left\|\mathbf{M}_{1}^{D}\left(\zeta, x_{0}, \alpha_{0}\right)-\mathbf{M}_{2}^{D}\left(\zeta, x_{0}, \alpha_{0}\right)\right\|_{\mathbb{C}^{2 m \times 2 m}} \underset{\substack{|\zeta| \rightarrow \infty \\ \zeta \in \rho_{\theta / 2}}}{=} O\left(\operatorname{Im}(\zeta) e^{-2 \operatorname{Im}(\zeta) a}\right) .
$$

Together, (4.84) and (4.88) yield (4.80).

Assuming (4.81), in order to prove (4.82), it suffices to prove

$$
\left\|\mathbf{M}_{1}^{D}\left(\zeta, x_{0}, \alpha_{0}\right)-\mathbf{M}_{2}^{D}\left(\zeta, x_{0}, \alpha_{0}\right)\right\|_{\mathbb{C}^{2 m \times 2 m}} \underset{\substack{\zeta \zeta \rightarrow \infty \\ \zeta \in \rho_{\theta_{\ell} / 2}}}{=} O\left(e^{-2 \operatorname{Im}(\zeta)(a-\epsilon)}\right), \quad \epsilon>0, \ell=1,2,
$$

since $\rho_{\theta_{1} / 2}$ (resp., $\rho_{\theta_{2} / 2}$ ) is a ray in $\mathbb{C}$ with $0<\arg (\zeta)=\theta_{1} / 2<\pi / 2$ (resp., $\left.\pi / 2<\arg (\zeta)=\theta_{2} / 2<\pi\right)$. Indeed, by [16, Theorem 5.5], (4.89) implies

$$
B_{1}(x)=B_{2}(x) \text { for a.e. } x \in\left[x_{0}-a, x_{0}+a\right],
$$

which clearly yields (4.82). In order to prove (4.89), let $\epsilon>0$. Making use of (4.35), one writes

$$
\begin{aligned}
& \left\|\mathbf{M}_{1}^{D}\left(\zeta, x_{0}, \alpha_{0}\right)-\mathbf{M}_{2}^{D}\left(\zeta, x_{0}, \alpha_{0}\right)\right\|_{\mathbb{C}^{2 m \times 2 m}} \\
& \quad=\left\|\left(\begin{array}{cc}
\zeta I_{m} & 0 \\
0 & I_{m}
\end{array}\right)\left[\widehat{\mathbf{M}}_{1,1}\left(z, x_{0}\right)-\widehat{\mathbf{M}}_{1,2}\left(z, x_{0}\right)\right]\left(\begin{array}{cc}
I_{m} & 0 \\
0 & \zeta^{-1} I_{m}
\end{array}\right)\right\|_{\mathbb{C}^{2 m \times 2 m}} \\
& \quad \leqslant C|\zeta|\left\|\widehat{\mathbf{M}}_{1,1}\left(z, x_{0}\right)-\widehat{\mathbf{M}}_{1,2}\left(z, x_{0}\right)\right\|_{\mathbb{C}^{2 m \times 2 m}}, \quad z=\zeta^{2}, \zeta \in \rho_{\theta_{\ell} / 2},|\zeta| \geqslant 1, \ell=1,2,
\end{aligned}
$$

for some $\zeta$-independent constant $C>0$. Choosing $\varepsilon=2 \epsilon$, one computes

$$
\begin{aligned}
& e^{2 \operatorname{Im}(\zeta)(a-\epsilon)}\left\|\mathbf{M}_{1}^{D}\left(\zeta, x_{0}, \alpha_{0}\right)-\mathbf{M}_{2}^{D}\left(\zeta, x_{0}, \alpha_{0}\right)\right\|_{\mathbb{C}^{2 m \times 2 m}} \\
& \quad \leqslant C|\zeta| e^{2 \operatorname{Im}(\zeta)(a-\epsilon)}\left\|\widehat{\mathbf{M}}_{1,1}\left(\zeta^{2}, x_{0}\right)-\widehat{\mathbf{M}}_{1,2}\left(\zeta^{2}, x_{0}\right)\right\|_{\mathbb{C}^{2 m \times 2 m}} \\
& \quad \leqslant \widetilde{C} \operatorname{Im}(\zeta) e^{2 \operatorname{Im}(\zeta)(a-\epsilon)} e^{-2 \operatorname{Im}(\zeta)(a-\varepsilon)} \\
& \quad=\widetilde{C} \operatorname{Im}(\zeta) e^{-2 \operatorname{Im}(\zeta) \epsilon}, \quad \zeta \in \rho_{\theta_{\ell} / 2},|\zeta| \gg 1, \ell=1,2,
\end{aligned}
$$

where $\tilde{C}>0$ is an appropriate $\zeta$-independent constant. The estimate in (4.92) makes use of (4.91) and the estimate in (4.93) uses the assumption in (4.81) which is applicable since $\zeta \in \rho_{\theta_{\ell} / 2},|\zeta| \gg 1$ implies $z=\zeta^{2} \in \rho_{\theta_{\ell}},|z| \gg 1$. The function of $\zeta$ in (4.94) is bounded as $|\zeta| \rightarrow \infty, \zeta \in \rho_{\theta_{\ell} / 2}, \ell=1,2$; hence, (4.94) implies

$$
\left\|\mathbf{M}_{1}^{D}\left(\zeta, x_{0}, \alpha_{0}\right)-\mathbf{M}_{2}^{D}\left(\zeta, x_{0}, \alpha_{0}\right)\right\|_{\mathbb{C}^{2 m \times 2 m}} \underset{\substack{|\zeta| \rightarrow \infty \\ \zeta \in \rho_{\theta_{\ell} / 2}}}{=} O\left(e^{-2 \operatorname{Im}(\zeta)(a-\epsilon)}\right), \quad \ell=1,2 .
$$

Since $\epsilon>0$ was arbitrary, (4.89) is established.

The reason for the additional assumption $\phi_{j} \in L^{\infty}\left(\left[x_{0}-a, x_{0}+a\right]\right)^{m \times m}, j=1,2$, in the case $m>1$, is due to a technical issue as explained in detail in 16. Remark 5.4]. It should be noted in this connection that for matrix-valued Schrödinger operators $H_{1, j}, j=1,2$, with the standard hypothesis on potentials, that is, $V_{j}=$ $V_{j}^{*} \in L_{\text {loc }}^{1}(\mathbb{R})^{m \times m}$, the local Borg-Marchenko-type results in Theorem 4.8 were 
derived in 41] (see also [45]). In this case no analog of the local boundedness assumptions on $\phi_{j}$ were necessary.

Finally, in the context of Theorem 4.8, we would also like to point out that an alternative approach to local Borg-Marchenko-type uniqueness results for Diractype operators, including a procedure for recovering the potential coefficient, was developed by Sakhnovich [118] (see also [119] and [120, Sects. 2.2.3, 4.2.2]).

\section{Appendix A. Supersymmetric Dirac-Type Operators in a Nutshell}

In this appendix we briefly summarize some results on supersymmetric Diractype operators and commutation methods due to [23, [43], [133, and [134, Ch. 5] (see also [55]).

The standing assumption in this appendix will be the following.

Hypothesis A.1. Let $\mathcal{H}_{j}, j=1,2$, be separable complex Hilbert spaces and

$$
T: \mathcal{H}_{1} \supseteq \operatorname{dom}(T) \rightarrow \mathcal{H}_{2}
$$

be a densely defined, closed, linear operator.

We define the self-adjoint Dirac-type operator in $\mathcal{H}_{1} \oplus \mathcal{H}_{2}$ by

$$
Q=\left(\begin{array}{cc}
0 & T^{*} \\
T & 0
\end{array}\right), \quad \operatorname{dom}(Q)=\operatorname{dom}(T) \oplus \operatorname{dom}\left(T^{*}\right) .
$$

Operators of the type $Q$ play a role in supersymmetric quantum mechanics (see, e.g., the extensive list of references in [13]). Then,

$$
Q^{2}=\left(\begin{array}{cc}
T^{*} T & 0 \\
0 & T T^{*}
\end{array}\right)
$$

and for notational purposes we also introduce

$$
H_{1}=T^{*} T \text { in } \mathcal{H}_{1}, \quad H_{2}=T T^{*} \text { in } \mathcal{H}_{2} .
$$

In the following, we also need the polar decomposition of $T$ and $T^{*}$, that is, the representations

$$
\begin{aligned}
T & =V_{T}|T|=\left|T^{*}\right| V_{T}=V_{T} T^{*} V_{T} \text { on } \operatorname{dom}(T)=\operatorname{dom}(|T|), \\
T^{*} & =V_{T^{*}}\left|T^{*}\right|=|T| V_{T^{*}}=V_{T^{*}} T V_{T^{*}} \text { on } \operatorname{dom}\left(T^{*}\right)=\operatorname{dom}\left(\left|T^{*}\right|\right), \\
|T| & =V_{T^{*}} T=T^{*} V_{T}=V_{T^{*}}\left|T^{*}\right| V_{T} \text { on } \operatorname{dom}(|T|), \\
\left|T^{*}\right| & =V_{T} T^{*}=T V_{T^{*}}=V_{T}|T| V_{T^{*}} \text { on } \operatorname{dom}\left(\left|T^{*}\right|\right),
\end{aligned}
$$

where

$$
\begin{aligned}
& |T|=\left(T^{*} T\right)^{1 / 2}, \quad\left|T^{*}\right|=\left(T T^{*}\right)^{1 / 2}, \quad V_{T^{*}}=\left(V_{T}\right)^{*}, \\
& V_{T^{*}} V_{T}=P \overline{\operatorname{ran}(|T|)}=P \overline{\operatorname{ran}\left(T^{*}\right)}, \quad V_{T} V_{T^{*}}=P_{\overline{\operatorname{ran}\left(\left|T^{*}\right|\right)}}=P \overline{\operatorname{ran}(T)} .
\end{aligned}
$$

In particular, $V_{T}$ is a partial isometry with initial set $\overline{\operatorname{ran}(|T|)}$ and final set $\overline{\operatorname{ran}(T)}$ and hence $V_{T^{*}}$ is a partial isometry with initial set $\overline{\operatorname{ran}\left(\left|T^{*}\right|\right)}$ and final set $\overline{\operatorname{ran}\left(T^{*}\right)}$. In addition,

$$
V_{T}= \begin{cases}\overline{T\left(T^{*} T\right)^{-1 / 2}}=\overline{\left(T T^{*}\right)^{-1 / 2} T} & \text { on }(\operatorname{ker}(T))^{\perp}, \\ 0 & \text { on } \operatorname{ker}(T) .\end{cases}
$$

Next, we collect some properties relating $H_{1}$ and $H_{2}$. 
Theorem A.2 (23]). Assume Hypothesis A.1 and let $\phi$ be a bounded Borel measurable function on $\mathbb{R}$.

(i) One has

$$
\begin{aligned}
& \operatorname{ker}(T)=\operatorname{ker}\left(H_{1}\right)=\left(\operatorname{ran}\left(T^{*}\right)\right)^{\perp}, \quad \operatorname{ker}\left(T^{*}\right)=\operatorname{ker}\left(H_{2}\right)=(\operatorname{ran}(T))^{\perp}, \\
& V_{T} H_{1}^{n / 2}=H_{2}^{n / 2} V_{T}, n \in \mathbb{N}, \quad V_{T} \phi\left(H_{1}\right)=\phi\left(H_{2}\right) V_{T} .
\end{aligned}
$$

(ii) $H_{1}$ and $H_{2}$ are essentially isospectral, that is,

$$
\sigma\left(H_{1}\right) \backslash\{0\}=\sigma\left(H_{2}\right) \backslash\{0\},
$$

in fact,

$$
T^{*} T\left[I_{\mathcal{H}_{1}}-P_{\operatorname{ker}(T)}\right] \text { is unitarily equivalent to } T T^{*}\left[I_{\mathcal{H}_{2}}-P_{\operatorname{ker}\left(T^{*}\right)}\right] \text {. }
$$

In addition,

$$
\begin{aligned}
& f \in \operatorname{dom}\left(H_{1}\right) \text { and } H_{1} f=\lambda^{2} f, \lambda \neq 0, \\
& \quad \text { implies } T f \in \operatorname{dom}\left(H_{2}\right) \text { and } H_{2}(T f)=\lambda^{2}(T f), \\
& g \in \operatorname{dom}\left(H_{2}\right) \text { and } H_{2} g=\mu^{2} g, \mu \neq 0, \\
& \quad \text { implies } T^{*} g \in \operatorname{dom}\left(H_{1}\right) \text { and } H_{1}\left(T^{*} g\right)=\mu^{2}\left(T^{*} g\right),
\end{aligned}
$$

with multiplicities of eigenvalues preserved.

(iii) One has for $z \in \rho\left(H_{1}\right) \cap \rho\left(H_{2}\right)$,

$$
\begin{aligned}
I_{\mathcal{H}_{2}}+z\left(H_{2}-z I_{\mathcal{H}_{2}}\right)^{-1} & \supseteq T\left(H_{1}-z I_{\mathcal{H}_{1}}\right)^{-1} T^{*}, \\
I_{\mathcal{H}_{1}}+z\left(H_{1}-z I_{\mathcal{H}_{1}}\right)^{-1} & \supseteq T^{*}\left(H_{2}-z I_{\mathcal{H}_{2}}\right)^{-1} T,
\end{aligned}
$$

and

$$
\begin{aligned}
& T^{*} \phi\left(H_{2}\right) \supseteq \phi\left(H_{1}\right) T^{*}, \quad T \phi\left(H_{1}\right) \supseteq \phi\left(H_{2}\right) T, \\
& V_{T^{*}} \phi\left(H_{2}\right) \supseteq \phi\left(H_{1}\right) V_{T^{*}}, \quad V_{T} \phi\left(H_{1}\right) \supseteq \phi\left(H_{2}\right) V_{T} .
\end{aligned}
$$

As noted by E. Nelson (unpublished), Theorem A.2 follows from the spectral theorem and the elementary identities,

$$
\begin{aligned}
& Q=V_{Q}|Q|=|Q| V_{Q}, \\
& \operatorname{ker}(Q)=\operatorname{ker}(|Q|)=\operatorname{ker}\left(Q^{2}\right)=(\operatorname{ran}(Q))^{\perp}=\operatorname{ker}(T) \oplus \operatorname{ker}\left(T^{*}\right), \\
& I_{\mathcal{H}_{1} \oplus \mathcal{H}_{2}}+z\left(Q^{2}-z I_{\mathcal{H}_{1} \oplus \mathcal{H}_{2}}\right)^{-1}=Q^{2}\left(Q^{2}-z I_{\mathcal{H}_{1} \oplus \mathcal{H}_{2}}\right)^{-1} \supseteq Q\left(Q^{2}-z I_{\mathcal{H}_{1} \oplus \mathcal{H}_{2}}\right)^{-1} Q, \\
& \\
& Q \phi\left(Q^{2}\right) \supseteq \phi\left(Q^{2}\right) Q,
\end{aligned}
$$

where

$$
V_{Q}=\left(\begin{array}{cc}
0 & \left(V_{T}\right)^{*} \\
V_{T} & 0
\end{array}\right)=\left(\begin{array}{cc}
0 & V_{T^{*}} \\
V_{T} & 0
\end{array}\right)
$$

In particular,

$$
\operatorname{ker}(Q)=\operatorname{ker}(T) \oplus \operatorname{ker}\left(T^{*}\right), \quad P_{\operatorname{ker}(Q)}=\left(\begin{array}{cc}
P_{\operatorname{ker}(T)} & 0 \\
0 & P_{\operatorname{ker}\left(T^{*}\right)}
\end{array}\right),
$$

and we also recall that

$$
\mathfrak{S}_{3} Q \mathfrak{S}_{3}=-Q, \quad \mathfrak{S}_{3}=\left(\begin{array}{cc}
I_{\mathcal{H}_{1}} & 0 \\
0 & -I_{\mathcal{H}_{2}}
\end{array}\right)
$$


that is, $Q$ and $-Q$ are unitarily equivalent. (For more details on Nelson's trick see also [132, Sect. 8.4], [134, Subsect. 5.2.3].) We also note that

$$
\psi(|Q|)=\left(\begin{array}{cc}
\psi(|T|) & 0 \\
0 & \psi\left(\left|T^{*}\right|\right)
\end{array}\right)
$$

for Borel measurable functions $\psi$ on $\mathbb{R}$, and

$$
\overline{\left[Q|Q|^{-1}\right]}=\left(\begin{array}{cc}
0 & \left(V_{T}\right)^{*} \\
V_{T} & 0
\end{array}\right)=V_{Q} \text { if } \operatorname{ker}(Q)=\{0\} .
$$

Finally, we recall the following relationships between $Q$ and $H_{j}, j=1,2$.

Theorem A.3 ([13, [133). Assume Hypothesis A.1.

(i) Introducing the unitary operator $U$ on $(\operatorname{ker}(Q))^{\perp}$ by

$$
U=2^{-1 / 2}\left(\begin{array}{cc}
I_{\mathcal{H}_{1}} & \left(V_{T}\right)^{*} \\
-V_{T} & I_{\mathcal{H}_{2}}
\end{array}\right) \text { on }(\operatorname{ker}(Q))^{\perp}
$$

one infers that

$$
U Q U^{-1}=\left(\begin{array}{cc}
|A| & 0 \\
0 & -\left|A^{*}\right|
\end{array}\right) \text { on }(\operatorname{ker}(Q))^{\perp}
$$

(ii) One has

$$
\begin{aligned}
\left(Q-\zeta I_{\mathcal{H}_{1} \oplus \mathcal{H}_{2}}\right)^{-1}=\left(\begin{array}{cc}
\zeta\left(H_{1}-\zeta^{2} I_{\mathcal{H}_{1}}\right)^{-1} & T^{*}\left(H_{2}-\zeta^{2} I_{\mathcal{H}_{2}}\right)^{-1} \\
T\left(H_{1}-\zeta^{2} I_{\mathcal{H}_{1}}\right)^{-1} & \zeta\left(H_{2}-\zeta^{2} I_{\mathcal{H}_{2}}\right)^{-1}
\end{array}\right), \\
\zeta^{2} \in \rho\left(H_{1}\right) \cap \rho\left(H_{2}\right) .
\end{aligned}
$$

(iii) In addition,

$$
\begin{aligned}
& \left(\begin{array}{c}
f_{1} \\
f_{2}
\end{array}\right) \in \operatorname{dom}(Q) \text { and } Q\left(\begin{array}{l}
f_{1} \\
f_{2}
\end{array}\right)=\eta\left(\begin{array}{l}
f_{1} \\
f_{2}
\end{array}\right), \eta \neq 0, \\
& \quad \text { implies } f_{j} \in \operatorname{dom}\left(H_{j}\right) \text { and } H_{j} f_{j}=\eta^{2} f_{j}, j=1,2 .
\end{aligned}
$$

Conversely,

$$
\begin{aligned}
& f \in \operatorname{dom}\left(H_{1}\right) \text { and } H_{1} f=\lambda^{2} f, \lambda \neq 0 \\
& \quad \text { implies }\left(\begin{array}{c}
f \\
\lambda^{-1} T f
\end{array}\right) \in \operatorname{dom}(Q) \text { and } Q\left(\begin{array}{c}
f \\
\lambda^{-1} T f
\end{array}\right)=\lambda\left(\begin{array}{c}
f \\
\lambda^{-1} T f
\end{array}\right) .
\end{aligned}
$$

Similarly,

$$
\begin{aligned}
& g \in \operatorname{dom}\left(H_{2}\right) \text { and } H_{2} g=\mu^{2} g, \mu \neq 0, \\
& \quad \text { implies }\left(\begin{array}{c}
\mu^{-1} T^{*} g \\
g
\end{array}\right) \in \operatorname{dom}(Q) \text { and } Q\left(\begin{array}{c}
\mu^{-1} T^{*} g \\
g
\end{array}\right)=\mu\left(\begin{array}{c}
\mu^{-1} T^{*} g \\
g
\end{array}\right) .
\end{aligned}
$$

Acknowledgments. We are indebted to Rostyk Hryniv and Alexander Sakhnovich for very helpful discussions.

\section{REFERENCES}

[1] S. Albeverio, F. Gesztesy, R. Høegh-Krohn, and H. Holden, Solvable Models in Quantum Mechanics, 2nd ed., AMS-Chelsea Publ., Providence, RI, 2005.

[2] S. Albeverio, R. Hryniv, and Ya. Mykytyuk, On spectra of non-self-adjoint Sturm-Liouville operators, Sel. Math. New Ser. 13, 571-599 (2008).

[3] S. Albeverio, A. Kostenko, and M. Malamud, Spectral theory of semibounded Sturm-Liouville operators with local interactions on a discrete set, J. Math. Phys. 51, 102102 (2010), 24pp. 
[4] S. Albeverio and P. Kurasov, Singular Perturbations of Differential Operators, Cambridge Univ. Press, Cambridge, 2001.

[5] G. D. Allen and F. J. Narcowich, R-operators I. Representation Theory and Applications, Indiana Univ. Math. J. 25, 945-963 (1976).

[6] N. Aronszajn and W. F. Donoghue, On exponential representations of analytic functions in the upper half-plane with positive imaginary part, J. Analyse Math. 5, 321-388 (1956-57).

[7] M.-L. Baeteman and K. Chadan, The inverse scattering problem for singular oscillating potentials, Nuclear Phys. A 255, 35-44 (1975).

[8] M.-L. Baeteman and K. Chadan, Scattering theory with highly singular oscillating potentials, Ann. Inst. H. Poincaré Sect. A 24, 1-16 (1976).

[9] J.G. Bak and A. A. Shkalikov, Multipliers in dual Sobolev spaces and Schrödinger operators with distribution potentials, Math. Notes 71, 587-594 (2002).

[10] J. Ben Amara and A. A. Shkalikov, Oscillation theorems for Sturm-Liouville problems with distribution potentials, Moscow Univ. Math. Bull. 64, no. 3, 132-137 (2009).

[11] A. Ben Amor and C. Remling, Direct and inverse spectral theory of one-dimensional Schrödinger operators with measures, Integral Eq. Operator Th. 52, 395-417 (2005).

[12] C. Bennewitz and W. N. Everitt, On second-order left-definite boundary value problems, in Ordinary differential equations and operators (Dundee, 1982), Lecture Notes in Math., Vol. 1032, Springer, Berlin, 1983, pp. 31-67.

[13] D. Bollé, F. Gesztesy, H. Grosse, W. Schweiger, and B. Simon, Witten index, axial anomaly, and Krein's spectral shift function in supersymmetric quantum mechanics, J. Math. Phys. 28, 1512-1525 (1987).

[14] T. Buckmaster and H. Koch, The Korteweg-de Vries equation at $H^{-1}$ regularity, arXiv:1112.4657.

[15] R. W. Carey, A unitary invariant for pairs of self-adjoint operators, J. reine angew. Math. 283, 294-312 (1976).

[16] S. Clark and F. Gesztesy, Weyl-Titchmarsh $M$-function asymptotics and Borg-type theorems for Dirac operators, Trans. Amer. Math. Soc. 354, 3475-3534 (2002).

[17] S. Clark and F. Gesztesy, On Povzner-Wienholtz-type self-adjointness results for matrixvalued Sturm-Liouville operators, Proc. Roy. Soc. Edinburgh 133A, 747-758 (2003).

[18] S. Clark, F. Gesztesy, and M. Zinchenko, Weyl-Titchmarsh theory and Borg-Marchenko-type uniqueness results for CMV operators with matrix-valued Verblunsky coefficients, Operators and Matrices 1, 535-592 (2007).

[19] S. Clark, F. Gesztesy, and M. Zinchenko, Borg-Marchenko-type uniqueness results for CMV operators, Trans. Roy. Norw. Soc. Sci. Lett. 2008(1), 1-18.

[20] M. Combescure and J. Ginibre, Spectral and scattering theory for the Schrödinger operator with strongly oscillating potentials, Ann. Inst. H. Poincaré 24, 17-29 (1976).

[21] M. Combescure, Spectral and scattering theory for a class of strongly oscillating potentials, Commun. Math. Phys. 73, 43-62 (1980).

[22] E. B. Davies, Singular Schrödinger operators in one dimension, Mathematika 59, 141-159 (2013).

[23] P. A. Deift, Applications of a commutation formula, Duke Math. J. 45, 267-310 (1978).

[24] P. Djakov and B. Mityagin, Spectral gap asymptotics of one-dimensional Schrödinger operators with singular periodic potentials, Integral Transforms Special Fcts. 20, nos. 3-4, 265-273 (2009).

[25] P. Djakov and B. Mityagin, Spectral gaps of Schrödinger operators with periodic singular potentials, Dyn. PDE 6, no. 2, 95-165 (2009).

[26] P. Djakov and B. Mityagin, Fourier method for one-dimensional Schrödinger operators with singular periodic potentials, in Topics in Operator Theory, Vol. 2: Systems and Mathematical Physics, J. A. Ball, V. Bolotnikov, J. W. Helton, L. Rodman, I. M. Spitkovsky (eds.), Operator Theory: Advances and Applications, Vol. 203, Birhäuser, Basel, 2010, pp. 195-236.

[27] P. Djakov and B. Mityagin, Criteria for existence of Riesz bases consisting of root functions of Hill and 1d Dirac operators, J. Funct. Anal. 263, 2300-2332 (2012).

[28] J. Eckhardt, Inverse uniqueness results for Schrödinger operators using de Branges theory, Complex Anal. Operator Th. 8, 37-50 (2014).

[29] J. Eckhardt, F. Gesztesy, R. Nichols, and G. Teschl, Weyl-Titchmarsh theory for SturmLiouville operators with distributional potentials, Opuscula Math. 33, 467-563 (2013). 
[30] J. Eckhardt, F. Gesztesy, R. Nichols, and G. Teschl, Inverse spectral theory for SturmLiouville operators with distributional potentials, J. London Math. Soc. (2) 88, 801-828 (2013).

[31] J. Eckhardt and G. Teschl, Uniqueness results for one-dimensional Schrödinger operators with purely discrete spectra, Trans. Amer. Math. Soc. 365, 3923-3942 (2013).

[32] J. Eckhardt and G. Teschl, Sturm-Liouville operators with measure-valued coefficients, J. Analyse Math. 120, no. 1, 151-224 (2013).

[33] D. E. Edmunds and W. D. Evans, Spectral Theory and Differential Operators, Clarendon Press, Oxford, 1989.

[34] W. N. Everitt and L. Markus, Boundary Value Problems and Symplectic Algebra for Ordinary Differential and Quasi-Differential Operators, Math. Surv. and Monographs, Vol. 61, Amer. Math. Soc., RI, 1999.

[35] C. Frayer, R. O. Hryniv, Ya. V. Mykytyuk, and P. A. Perry, Inverse scattering for Schrödinger operators with Miura potentials: I. Unique Riccati representatives and ZS-AKNS systems, Inverse Probl. 25, 115007 (25pp) (2009).

[36] C. Fulton, Titchmarsh-Weyl m-functions for second order Sturm-Liouville problems, Math. Nachr. 281, 1417-1475 (2008).

[37] C. Fulton and H. Langer, Sturm-Liouville operators with singularities and generalized Nevanlinna functions, Complex Anal. Operator Th. 4, 179-243 (2010).

[38] C. Fulton, H. Langer, and A. Luger, Mark Krein's method of directing functionals and singular potentials, Math. Nachrichten 285, 1791-1798 (2012).

[39] F. Gesztesy, On the modified Korteweg-deVries equation, in Differential Equations with Applications in Biology, Physics, and Engineering, F. Kappel, J. A. Goldstein, and W. Schappacher (eds.), Marcel Dekker, 1991, p. 139-183.

[40] F. Gesztesy, Quasi-periodic, finite-gap solutions of the modified Korteweg-deVries equation, in Ideas and Methods in Mathematical Analysis, Stochastics, and Applications, Vol. 1, S. Albeverio, J. E. Fenstad, H. Holden, and T. Lindstrøm (eds.),Cambridge University Press, 1992, pp. 428-471.

[41] F. Gesztesy, A. Kiselev, and K. A. Makarov, Uniqueness results for matrix-valued Schrödinger, Jacobi, and Dirac-type operators, Math. Nachr. 239-240, 103-145 (2002).

[42] F. Gesztesy, R. Nowell, and W. Pötz, One-dimensional scattering theory for quantum systems with nontrivial spatial asymptotics, Diff. Integral Eq. 10, 521-546 (1997).

[43] F. Gesztesy, W. Schweiger, and B. Simon, Commutation methods applied to the mKdVequation, Trans. Amer. Math. Soc. 324, 465-525 (1991).

[44] F. Gesztesy and B. Simon, Constructing solutions of the mKdV-equation, J. Funct. Anal. 89, 53-60 (1990)

[45] F. Gesztesy and B. Simon, On local Borg-Marchenko uniqueness results, Commun. Math. Phys. 211, 273-287 (2000).

[46] F. Gesztesy, B. Simon, and G. Teschl, Spectral deformations of one-dimensional Schrödinger operators, J. Analyse Math. 70, 267-324 (1996).

[47] F. Gesztesy and E. Tsekanovskii, On matrix-valued Herglotz functions, Math. Nachr. 218, 61-138 (2000).

[48] F. Gesztesy and R. Weikard, Some remarks on the spectral problem underlying the CamassaHolm hierarchy, in Operator Theory in Harmonic and Non-commutative Analysis, IWOTA 12, J. A. Ball, M. A. Drietschel, A. F. M. ter Elst, P. Portal, and D. Potapov (eds.), Operator Theory: Advances and Applications, Vol. 240, Birkhäuser, Springer, Basel, 2014, pp. 137-188.

[49] F. Gesztesy, R. Weikard, and M. Zinchenko, On spectral theory for Schrödinger operators with operator-valued potentials, J. Diff. Eq. 255, no. 7, 1784-1827 (2013).

[50] F. Gesztesy and M. Zinchenko, On spectral theory for Schrödinger operators with strongly singular potentials, Math. Nachr. 279, 1041-1082 (2006).

[51] A. S. Goriunov and V. A. Mikhailets, Resolvent convergence of Sturm-Liouville operators with singular potentials, Math. Notes 87, no. 2, 287-292 (2010).

[52] A. Goriunov and V. Mikhailets, Regularization of singular Sturm-Liouville equations, Meth. Funct. Anal. Topology 16, no. 2, 120-130 (2010).

[53] S. Grudsky and A. Rybkin, On positive type initial profiles for the KdV equation, arXiv:1108.2314 Proc. Amer. Math. Soc. (to appear).

[54] O. Hald, Discontinuous inverse eigenvalue problems, Commun. Pure. Appl. Math. 37 539577 (1984). 
[55] V. Hardt, A. Konstantinov, and R. Mennicken, On the spectrum of the product of closed operators, Math. Nachr. 215, 91-102 (2000).

[56] J. Herczyński, On Schrödinger operators with distributional potentials, J. Operator Th. 21, 273-295 (1989).

[57] D. B. Hinton and A. Schneider, On the Titchmarsh-Weyl coefficients for singular SHermitian Systems I, Math. Nachr. 163, 323-342 (1993).

[58] D. B. Hinton and A. Schneider, On the Titchmarsh-Weyl coefficients for singular SHermitian Systems II, Math. Nachr. 185, 67-84 (1997).

[59] D. B. Hinton and J. K. Shaw, On Titchmarsh-Weyl $M(\lambda)$-functions for linear Hamiltonian systems, J. Diff. Eq. 40, 316-342 (1981).

[60] D. B. Hinton and J. K. Shaw, On the spectrum of a singular Hamiltonian system, Quaest. Math. 5, 29-81 (1982).

[61] D. B. Hinton and J. K. Shaw, Hamiltonian systems of limit point or limit circle type with both endpoints singular, J. Diff. Eq. 50, 444-464 (1983).

[62] D. B. Hinton and J. K. Shaw, On boundary value problems for Hamiltonian systems with two singular points, SIAM J. Math. Anal. 15, 272-286 (1984).

[63] D. B. Hinton and J. K. Shaw, On the spectrum of a singular Hamiltonian system, II, Quaest. Math. 10, 1-48 (1986).

[64] R. O. Hryniv, Analyticity and uniform stability in the inverse singular Sturm-Liouville spectral problem, Inverse Probl. 27, 065011 (2011), 25pp.

[65] R. O. Hryniv and Ya. V. Mykytyuk, $1 D$ Schrödinger operators with periodic singular potentials, Methods Funct. Anal. Topology 7, no. 4, 31-42 (2001).

[66] R. O. Hryniv and Ya. V. Mykytyuk, $1 D$ Schrödinger operators with singular Gordon potentials, Methods Funct. Anal. Topology 8, no. 1, 36-48 (2002).

[67] R. O. Hryniv and Ya. V. Mykytyuk, Inverse spectral problems for Sturm-Liouville operators with singular potentials, Inverse Probl. 19, 665-684 (2003).

[68] R. O. Hryniv and Ya. V. Mykytyuk, Half-inverse spectral problems for Sturm-Liouville operators with singular potentials, Inverse Probl. 20, 1423-1444 (2004).

[69] R. O. Hryniv and Ya. V. Mykytyuk, Transformation operators for Sturm-Liouville operators with singular potentials, Math. Phys. Anal. Geom. 7, 119-149 (2004).

[70] R. O. Hryniv and Ya. V. Mykytyuk, Inverse spectral problems for Sturm-Liouville operators with singular potentials. IV. Potentials in the Sobolev space scale, Proc. Edinburgh Math. Soc. (2) 49, 309-329 (2006).

[71] R. O. Hryniv and Ya. V. Mykytyuk, Eigenvalue asymptotics for Sturm-Liouville operators with singular potentials, J. Funct. Anal. 238, 27-57 (2006).

[72] R. O. Hryniv and Ya. V. Mykytyuk, Self-adjointness of Schrödinger operators with singular potentials, Meth. Funct. Anal. Topology 18, 152-159 (2012).

[73] R. O. Hryniv, Ya. V. Mykytyuk, and P. A. Perry, Inverse scattering for Schrödinger operators with Miura potentials: II. Different Riccati representatives, Commun. Part. Diff. Eq. 36, 1587-1623 (2011).

[74] R. O. Hryniv, Ya. V. Mykytyuk, and P. A. Perry, Sobolev mapping properties of the scattering transform for the Schrödinger equation, in "Spectral Theory and Geometric Analysis", M. Braverman, L. Friedlander, T. Kappeler, P. Kuchment, P. Topalov, and J. Weitsman (eds.), Contemp. Math. 535, 79-93 (2011).

[75] I. S. Kac, The existence of spectral functions of generalized second order differential systems with boundary conditions at the singular end, Transl. Amer. Math. Soc., Ser. 2, 62, 204-262 (1967).

[76] T. Kappeler and C. Möhr, Estimates for periodic and Dirichlet eigenvalues of the Schrödinger operator with singular potentials, J. Funct. Anal. 186, 62-91 (2001).

[77] T. Kappeler, P. Perry, M. Shubin, and P. Topalov, The Miura map on the line, Int. Math. Res. Notices, 2005, no. 50, 3091-3133.

[78] T. Kappeler and P. Topalov, Global fold structure of he Miura map on $L^{2}(\mathbb{T})$, Int. Math. Res. Notices, 2004, no. 39, 2039-2068.

[79] T. Kappeler and P. Topalov, Global well-posedness of $m K d V$ in $L^{2}(\mathbb{T}, \mathbb{R})$, Commun. Part. Diff. Eq. 30, 435-449 (2005).

[80] T. Kappeler and P. Topalov, Global wellposedness of $K d V$ in $H^{-1}(\mathbb{T}, \mathbb{R})$, Duke Math. J. 135, 327-360 (2006). 
[81] M. Kato, Estimates of the eigenvalues of Hill's operators with distributional coefficients, Tokyo J. Math. 33, 361-364 (2010).

[82] T. Kato, Perturbation Theory for Linear Operators, corr. printing of the 2nd ed., Springer, Berlin, 1980.

[83] K. Kodaira, The eigenvalue problem for ordinary differential equations of the second order and Heisenberg's theory of S-matrices, Amer. J. Math. 71, 921-945 (1949).

[84] E. Korotyaev, Characterization of the spectrum of Schrödinger operators with periodic distributions, Int. Math. Res. Notices 2003, no. 37, 2019-2031.

[85] E. Korotyaev, Sharp asymptotics of the quasimomentum, Asymptot. Anal. 80, 269-287 (2012).

[86] A. S. Kostenko and M. M. Malamud, One-dimensional Schrödinger operator with $\delta$ interactions, Funct. Anal. Appl. 44, no. 2, 151-155 (2010).

[87] A. S. Kostenko and M. M. Malamud, 1-D Schrödinger operators with local point interactions on a discrete set, J. Diff. Eq. 249, 253-304 (2010).

[88] A. Kostenko, A. Sakhnovich, and G. Teschl, Inverse eigenvalue problems for perturbed spherical Schrödinger operators, Inverse Problems 26, 105013, 14pp (2010).

[89] A. Kostenko, A. Sakhnovich, and G. Teschl, Weyl-Titchmarsh theory for Schrödinger operators with strongly singular potentials, Int. Math. Res. Notices 2012, no. 8, 1699-1747 (2012).

[90] A. Kostenko, A. Sakhnovich, and G. Teschl, Commutation methods for Schrödinger operators with strongly singular potentials, Math. Nachr. 285, 392-410 (2012).

[91] A. Kostenko and G. Teschl, On the singular Weyl-Titchmarsh function of perturbed spherical Schrödinger operators, J. Diff. Eq. 250, 3701-3739 (2011).

[92] A. Kostenko and G. Teschl, Spectral asymptotics for perturbed spherical Schrödinger operators and applications to quantum scattering, arXiv:1205.5049 Commun. Math. Phys. 322, 255-275 (2013).

[93] S. Kotani and B. Simon, Stochastic Schrödinger operators and Jacobi matrices on the strip, Commun. Math. Phys. 119, 403-429 (1988).

[94] P. Kurasov and A. Luger, An operator theoretic interpretation of the generalized TitchmarshWeyl coefficient for a singular Sturm-Liouville problem, Math. Phys. Anal. Geom. 14, 115151 (2011).

[95] M. Lesch and M. Malamud, The inverse spectral problem for first order systems on the half line, Operator Theory: Advances and Applications, Vol. 117, Birkhäuser, Basel, 2000, p. 199-238.

[96] M. Lesch and M. Malamud, On the deficiency indices and self-adjointness of symmetric Hamiltonian systems, J. Diff. Eq. 189, 556-615 (2003).

[97] B. M. Levitan and M. Otelbaev, Conditions for self-adjointness of the Schrödinger and Dirac operators, Trans. Moscow. Math. Soc. 42, 139-156 (1982).

[98] V. G. Maz'ya and T. O. Shaposhnikova, Theory of Sobolev Multipliers. With Applications to Differential and Integral Operators, Springer, Berlin, 2009.

[99] V. G. Maz'ya and I. E. Verbitsky, Boundedness and compactness criteria for the onedimensional Schrödinger operator, in Function Spaces, Interpolation Theory and Related Topics, de Gruyter, Berlin, 2002, pp. 369-382.

[100] V. G. Maz'ya and I. E. Verbitsky, The Schrödinger operator on the energy space: boundedness and compactness criteria, Acta Math. 188, 263-302 (2002).

[101] V. G. Maz'ya and I. E. Verbitsky, Infinitesimal form boundedness and Trudinger's subordination for the Schrödinger operator, Invent. Math. 162, 81-136 (2005).

[102] V. G. Maz'ya and I. E. Verbitsky, Form boundedness of the general second-order differential operator, Commun. Pure Appl. Math. 59, 1286-1329 (2006).

[103] V. A. Mikhailets and V. M. Molyboga, Singular eigenvalue problems on the circle, Meth. Funct. Anal. Topology 10, no. 3, 44-53 (2004)

[104] V. A. Mikhailets and V. M. Molyboga, Uniform estimates for the semi-periodic eigenvalues of the singular differential operators, Meth. Funct. Anal. Topology 10, no. 4, 30-57 (2004).

[105] V. A. Mikhailets and V. M. Molyboga, Singularly perturbed periodic and semiperiodic differential operators, Ukrainian Math. J. 59, no. 6, 858-873 (2007).

[106] V. A. Mikhailets and V. M. Molyboga, One-dimensional Schrödinger operators with singular periodic potentials, Meth. Funct. Anal. Topology 14, no. 2, 184-200 (2008). 
[107] V. A. Mikhailets and V. M. Molyboga, Spectral gaps of the one-dimensional Schrödinger operators with singular periodic potentials, Meth. Funct. Anal. Topology 15, no. 1, 31-40 (2009).

[108] V. A. Mikhailets and V. M. Molyboga, Remarks on Schrödinger operators with singular matrix potentials, arXiv:1306.0439

[109] K. A. Mirzoev and T. A. Safanova, Singular Sturm-Liouville operators with distribution potential on spaces of vector functions, Dokl. Math. 84, 791-794 (2011).

[110] Ya. V. Mykytyuk and N. S. Trush, Inverse spectral problems for Sturm-Liouville operators with matrix-valued potentials, Inverse Probl. 26, 015009 (2010), 36pp.

[111] M. A. Naimark, Linear Differential Operators, Part II, F. Ungar, New York, 1968.

[112] D. B. Pearson, Scattering theory for a class of oscillating potentials, Helv. Phys. Acta 52, 541-5554 (1979).

[113] F. S. Rofe-Beketov and E. H. Hristov, Transformation operators and scattering functions for a highly singular potential, Sov. Math. Dokl. 7, 834-837 (1966).

[114] F. S. Rofe-Beketov and E. H. Hristov, Some analytical questions and the inverse SturmLiouville problem for an equation with highly singular potential, Sov. Math. Dokl. 10, 432-435 (1969).

[115] A. Rybkin, Regularized perturbation determinants and KdV conservation laws for irregular initial profiles, in Topics in Operator Theory. Vol. 2. Systems and Mathematical Physics, J. A. Ball, V. Bolotnikov, J. W. Helton, L. Rodman, I. M. Spitkovsky (eds.), Operator Theory: Advances and Applications, Vol. 203, Birkhäuser Verlag, Basel, 2010, 427-444pp.

[116] I. V. Sadovnichaya, Equiconvergence of expansions in series in eigenfunctions of SturmLiouville operators with distribution potentials, Sborn. Math. 201, 1307-1322 (2010).

[117] I. V. Sadovnichaya, Equiconvergence in Sobolev and Hölder spaces of expansions in eigenfunctions of Sturm-Liouville operators with distribution potentials, Dokl. Math. 83, 169-170 (2011).

[118] A. Sakhnovich, Dirac type and canonical systems: spectral and Weyl-Titchmarsh matrix functions, direct and inverse problems, Inverse Probl. 18, 331-348 (2002).

[119] A. Sakhnovich, Skew-self-adjoint discrete and continuous Dirac-type systems: inverse problems and Borg-Marchenko theorems, Inverse Problems 22, 2083-2101 (2006).

[120] A. L. Sakhnovich, L. A. Sakhnovich, and I. Ya. Roitberg, Inverse Problems and Nonlinear Evolution Equations. Solutions, Darboux Matrices and Weyl-Titchmarsh Functions, Studies in Mathematics, Vol. 47, De Gruyter, Berlin, 2013.

[121] A. M. Savchuk and A. A. Shkalikov, Sturm-Liouville operators with singular potentials, Math. Notes 66, 741-753 (1999).

[122] A. M. Savchuk and A. A. Shkalikov, The trace formula for Sturm-Liouville operators with singular potentials, Math. Notes 69, no. 3-4, 387-400 (2001).

[123] A. M. Savchuk and A. A. Shkalikov, Sturm-Liouville operators with distribution potentials, Trans. Moscow Math. Soc. 2003, 143-192.

[124] A. M. Savchuk and A. A. Shkalikov, Inverse problem for Sturm-Liouville operators with distribution potentials: reconstruction from two spectra, Russ. J. Math. Phys. 12, no. 4, 507-514 (2005).

[125] A. M. Savchuk and A. A. Shkalikov, On the eigenvalues of the Sturm-Liouville operator with potentials from Sobolev spaces, Math. Notes 80, 814-832 (2006).

[126] A. M. Savchuk and A. A. Shkalikov, On the properties of maps connected with inverse Sturm-Liouville problems, Proc. Steklov Inst. Math. 260, no. 1, 218-237 (2008).

[127] A. M. Savchuk and A. A. Shkalikov, Inverse problems for Sturm-Liouville operators with potentials in Sobolev spaces: uniform stability, Funct. Anal. Appl. 44, 270-285 (2010).

[128] M. Shahriari, A. Jodayree Akbarfam, and G. Teschl, Uniqueness for inverse Sturm-Liouville problems with a finite number of transmission conditions, J. Math. Anal. Appl. 395, 19-29 (2012).

[129] D. Shin, On Quasi-Differential Operators in Hilbert Space, Doklad. Akad. Nauk. SSSR 18, 523-526 (1938). (Russian.)

[130] D. Shin, On solutions of a linear quasi-differential equation of the nth order, Mat. Sbornik 7(49), 479-532 (1940). (Russian.)

[131] D. Shin, Quasi-differential operators in Hilbert space, Mat. Sbornik 13(55), 39-70 (1943). (Russian.) 
[132] G. Teschl, Mathematical Methods in Quantum Mechanics: With Applications to Schrödinger Operators, Graduate Studies in Mathematics, Amer. Math. Soc., Vol. 157, 2nd ed., RI, 2014.

[133] B. Thaller, Normal forms of an abstract Dirac operator and applications to scattering theory, J. Math. Phys. 29, 249-257 (1988).

[134] B. Thaller, The Dirac Equation, Springer, Berlin, 1992.

[135] J. Weidmann, Spectral Theory of Ordinary Differential Operators, Lecture Notes in Math., Vol. 1258, Springer, Berlin, 1987.

[136] A. Zettl, Formally self-adjoint quasi-differential operators, Rocky Mountain J. Math. 5, 453-474 (1975).

Faculty of Mathematics, University of Vienna, Oskar-Morgenstern-Platz 1, 1090 Wien, Austria

E-mail address: jonathan.eckhardt@univie.ac.at

$U R L:$ http://homepage.univie.ac.at/jonathan.eckhardt/

Department of Mathematics, University of Missouri, Columbia, MO 65211, USA

E-mail address: gesztesyf@missouri.edu

$U R L:$ http://www.math.missouri.edu/personnel/faculty/gesztesyf.html

Mathematics Department, The University of Tennessee at Chattanooga, 415 EMCS

Building, Dept. 6956, 615 McCallie Ave, Chattanooga, TN 37403, USA

E-mail address: Roger-Nichols@utc.edu

$U R L:$ http://www.utc.edu/faculty/roger-nichols/

Faculty of Mathematics, University of Vienna, Oskar-Morgenstern-Platz 1, 1090 Wien, Austria, and International Erwin Schrödinger Institute for Mathematical Physics, Boltzmanngasse 9, 1090 Wien, Austria

E-mail address: Gerald.Teschl@univie.ac.at

$U R L:$ http://www.mat.univie.ac.at/ gerald/ 